\title{
Microstructure and domain engineering of lithium niobate crystal films for integrated photonic applications
}

\author{
Dehui Sun', Yunwu Zhang ${ }^{1}$, Dongzhou Wang $\mathbb{C}^{2}$, Wei Song ${ }^{3}$, Xiaoyan Liu', Jinbo Pang ${ }^{1}$, Degiang Geng ${ }^{4}$, \\ Yuanhua Sang ${ }^{5}$ and Hong Liu Li, $^{1,5}$
}

\begin{abstract}
Recently, integrated photonics has attracted considerable interest owing to its wide application in optical communication and quantum technologies. Among the numerous photonic materials, lithium niobate film on insulator (LNOI) has become a promising photonic platform owing to its electro-optic and nonlinear optical properties along with ultralow-loss and high-confinement nanophotonic lithium niobate waveguides fabricated by the complementary metal-oxide-semiconductor (CMOS)-compatible microstructure engineering of LNOI. Furthermore, ferroelectric domain engineering in combination with nanophotonic waveguides on LNOI is gradually accelerating the development of integrated nonlinear photonics, which will play an important role in quantum technologies because of its ability to be integrated with the generation, processing, and auxiliary detection of the quantum states of light. Herein, we review the recent progress in CMOS-compatible microstructure engineering and domain engineering of LNOI for integrated lithium niobate photonics involving photonic modulation and nonlinear photonics. We believe that the great progress in integrated photonics on LNOI will lead to a new generation of techniques. Thus, there remains an urgent need for efficient methods for the preparation of LNOI that are suitable for large-scale and low-cost manufacturing of integrated photonic devices and systems.
\end{abstract}

\section{Introduction}

In contemporary society, the demand for highbandwidth optical communication, including for mobile high-definition video streaming, autonomous vehicle applications, remote surgery, telepresence applications, and interactive 3D virtual reality gaming, is sharp increasing ${ }^{1-5}$. The electro-optical modulator is the key component in optical fiber communication, which modulates the light signal for loading information through electricity. Lithium niobate $\left(\mathrm{LiNbO}_{3}, \mathrm{LN}\right)$ exhibits a highperformance electro-optic effect and high optical

Correspondence: Dehui Sun (ifc_sundh@ujn.edu.cn) or

Hong Liu (hongliu@sdu.edu.cn)

${ }^{1}$ Collaborative Innovation Center of Technology and Equipment for Biological Diagnosis and Therapy in Universities of Shandong, Institute for Advanced

Interdisciplinary Research (iAIR), University of Jinan, Jinan 250022, China

${ }^{2}$ Jinan Institute of Quantum Technology, Jinan 250101, China

Full list of author information is available at the end of the article transparency ${ }^{6,7}$. Therefore, $\mathrm{LiNbO}_{3}$ has been widely applied in electro-optic modulators. Generally, optical modulation is realized by a voltage-induced refractive index change, which can be described by the change in the ellipsoid of the refraction index influenced by an external electric field as $\Delta \beta_{i j}=\gamma_{i j k} E_{k}+h_{i j k l} E_{k} E_{l}+\ldots$, where $\Delta \beta_{i j}$ is the variation in the dielectric impermeability under the external electric field $(E), \gamma_{i j k}$ is the linear electro-optic coefficient or Pockels coefficient, and $h_{i j k l}$ is the quadratic electro-optic coefficient or Kerr coefficient. For LN, the linear electro-optic coefficient is $\gamma_{33}=30.9 \times 10^{-12} \mathrm{mV}^{-1}$, while other photonic materials usually have a very small or zero linear electro-optic coefficient. For example, the linear electro-optic coefficient of GaAs is $\gamma_{41}=1.5 \times$ $10^{-12} \mathrm{mV}^{-1}$, which is one-twentieth that of LN.

An electro-optic modulator can be constructed on LN wafers by fabricating waveguides via defect

\section{(c) The Author(s) 2020}

(c) (i) Open Access This article is licensed under a Creative Commons Attribution 4.0 International License, which permits use, sharing, adaptation, distribution and reproduction c. in any medium or format, as long as you give appropriate credit to the original author(s) and the source, provide a link to the Creative Commons license, and indicate if changes were made. The images or other third party material in this article are included in the article's Creative Commons license, unless indicated otherwise in a credit line to the material. If material is not included in the article's Creative Commons license and your intended use is not permitted by statutory regulation or exceeds the permitted use, you will need to obtain permission directly from the copyright holder. To view a copy of this license, visit http://creativecommons.org/licenses/by/4.0/. 


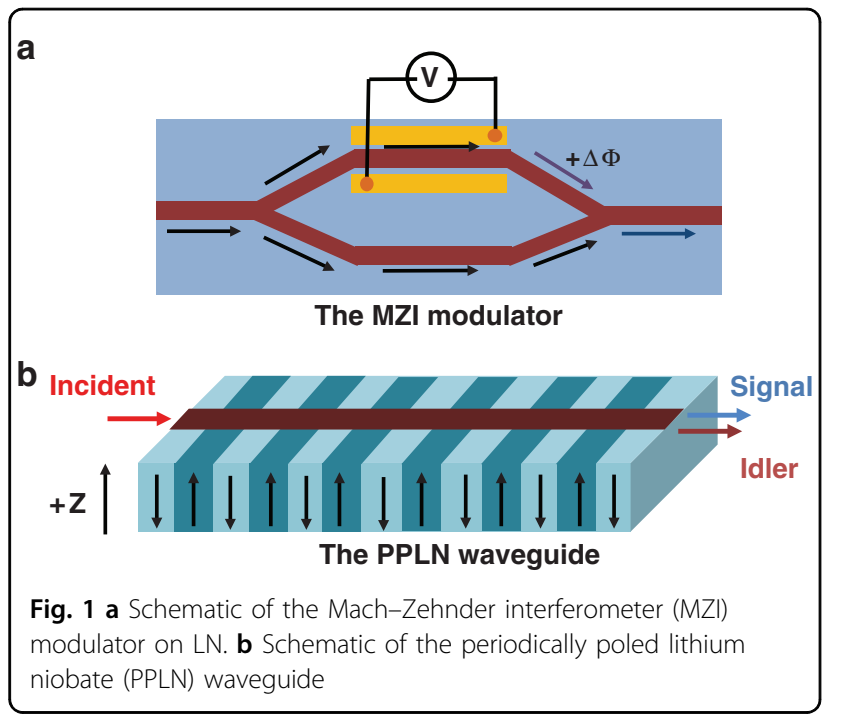

engineering, including titanium diffusion or proton exchange, which is compatible with complementary metal-oxide-semiconductor (CMOS) technology. For example, the 50:50 Y-junction microstructure splits the input light into two LN optical waveguides that form the two arms of the Mach-Zehnder interferometer (MZI) modulator, a traditional electric-optical modulator. As shown in Fig. 1a, the applied voltage induces a phase delay in one arm and a phase advance in the other, which in turn changes the output intensity at the Y-combiner through interference ${ }^{7}$. The minimum voltage required to completely switch the output between on and off is defined as the half-wave voltage $\left(V_{\pi}\right)$. However, the low refractive index contrast of the titanium diffusion $(\Delta n \sim 0.01)$ or proton exchange $(\Delta n$ $\sim 0.1$ ) waveguides resulted in weak confinement and a large bending radius of the waveguides ${ }^{8}$. The weak optical confinement requires metal electrodes to be placed far from the optical waveguide, which in turn lowers the electro-optic efficiency. Hence, either large voltages or long electrodes are required to sufficiently modulate traditional LN modulators (with a size of approximately several centimeters). Therefore, the product of the half-wave voltage and electrode length, $V_{\pi} L$, is a common figure of merit for electro-optic modulators. Nevertheless, the capacitance of the long electrodes limits the bandwidth, which results in a trade-off between the driving voltage and bandwidth ${ }^{7}$.

On the other hand, ferroelectric domain engineering of LN crystals has been extended from $1 \mathrm{D}$ to $2 \mathrm{D}$ and $3 \mathrm{D}$, which was comprehensively reviewed in reference ${ }^{9}$. Periodically poled lithium niobate (PPLN) has been widely applied in the fields of frequency conversion, nonlinear beam shaping ${ }^{10}$, and quantum entanglement based on quasi-phase-matching (QPM) theory ${ }^{11-15}$. The second- order nonlinear optical coefficients $\left(d_{33}\right.$ and $\left.d_{31}\right)$ of $\mathrm{LN}$ are 25.2 and $4.6 \mathrm{pm} \mathrm{V}^{-1}$ at $1064 \mathrm{~nm}$, respectively ${ }^{16}$. Because LN is only poled along the $z$-axis, PPLN is appropriate for polarizing light to parallel to the $z$-axis to exploit the largest nonlinear-optical coefficient $d_{33}$. As shown in Fig. 1b, a channel waveguide was fabricated on the surface of a PPLN wafer, which can confine the pump beam over the entire interaction length to greatly increase the conversion efficiency.

Moreover, considering the device size, reliability, cost, and energy consumption, photonic integrated circuit (PIC) technology has recently attracted significant interest in the rise of the integrated photonics field ${ }^{17-23}$. Integrated photonics is concerned with the integration of all the key components connected by a waveguide in a single chip (photonic platform) using a single material (monolithic integration) or multiple materials (hybrid integration). Most integrated photonic circuits have been built on four key platforms: indium phosphide ${ }^{24,25}$, silicon-oninsulator $(\mathrm{SOI})^{26-28}$, silicon nitride ${ }^{25,29}$, and $\mathrm{LN}^{30,31}$. Nevertheless, no photonic platform has yet produced an optimal overall photonics system performance ${ }^{17}$. Generally, the selection of a material platform is based on the functionality of the optical components in the circuit, a low propagation loss, and industry-compatible fabrication processes $^{18}$. For example, the compatibility of silicon integrated circuit manufacturing is the main reason for the development of silicon photonics ${ }^{32}$. Moreover, the manufacturing methods are suitable for the construction of SOI rib waveguides with a small cross-section of $1 \mu \mathrm{m}^{2}$ with propagation losses as low as $0.1-0.5 \mathrm{~dB} \mathrm{~cm}^{-1}$ and a high index contrast between the $\mathrm{Si}$ waveguide layers $\left(n_{\mathrm{Si}}\right.$ $\sim 3.4$ ) and $\mathrm{SiO}_{2}$ cladding layers $\left(n_{\mathrm{SiO} 2} \sim 1.4\right)$, affording strong optical confinement and a small bending radius of $10 \mu \mathrm{m}^{33}$. The low propagation loss and high index contrast of photonics systems, which make SOI one of the most active photonic platforms, are important evaluation standards for photonic platforms ${ }^{34}$. However, the $3 \mathrm{~dB}$ electrical modulation bandwidths of all-Si photonic devices have a theoretical limit of approximately $60 \mathrm{GHz}^{35}$.

To conclude, $\mathrm{LN}$ is a promising photonic matrix due to its advantageous electro-optical effect and nonlinear optical properties. For example, on-chip generation and manipulation of entangled photons based on annealed proton exchanged waveguide circuits integrated on a zcut PPLN crystal was demonstrated ${ }^{36}$. Unfortunately, bulk LN waveguides have large footprints, and the fabrication technology is not compatible with CMOS technology. Above all, nanophotonic LN waveguides with a larger refractive index contrast and a smaller optical mode size have emerged as a branch of integrated lithium niobate photonics $^{37-40}$, although LN was perceived as a difficultto-etch material. Over the past 25 years, integrated 


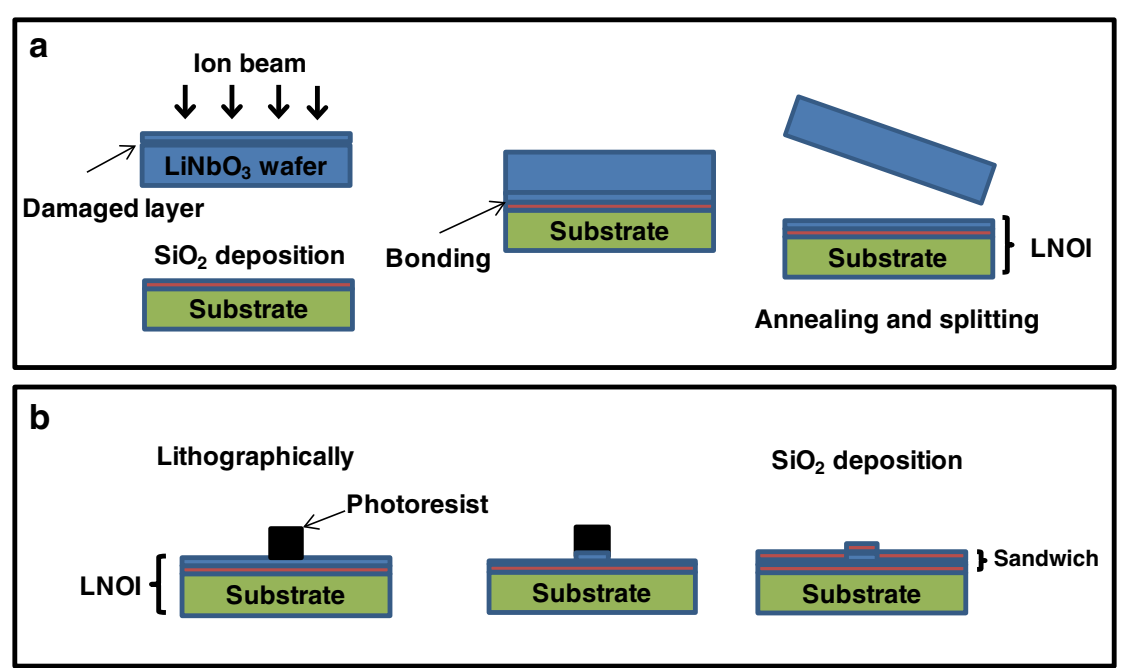

Fig. 2 a Schematic of the preparation of LNOI. b Schematic of the microstructure engineering of LNOI

lithium niobate photonics have relied almost exclusively on high-quality lithium niobate thin film on insulator (LNOI) technology ${ }^{41}$ and advanced PIC technology for etching nanophotonic waveguides and microphotonic modulators. On the one hand, LNOI with a diameter of 3 in. can be fabricated by the smart cut technique ${ }^{41}$. In this technique, a submicron single-crystalline LN film is first detached from a sacrificial wafer by crystal ion slicing and then bonded with a $\mathrm{SiO}_{2}$-deposited $\mathrm{LN}$ substrate or $\mathrm{Si}$ substrate, forming LNOI (Fig. 2a). On the other hand, the CMOS-compatible microstructure engineering of LNOI for integrated photonic circuits, including nanophotonic waveguides and advanced functional modulators, has advanced considerably. Briefly, the microstructure of integrated photonic circuits is transferred to LN thin films by a lithographically defined mask. Then, the LN thin film is etched by $\mathrm{Ar}^{+}$milling, forming thin slabs with a thickness of several hundred nanometers, which form ridge waveguides. Finally, a microscale-thick $\mathrm{SiO}_{2}$ cladding layer is deposited on top of the etched LN thin film by plasma-enhanced chemical vapor deposition (PECVD) to form a sandwich structure (Fig. 2b). The refractive index contrast between the $\mathrm{LN}$ core $\left(n_{\mathrm{LN}} \sim 2.1\right)$ and $\mathrm{SiO}_{2}$ cladding $\left(n_{\mathrm{SiO} 2} \sim 1.4\right)$ is approximately 0.7 , which is much larger than that of traditional LN waveguides; thus, LNOI can serve as strongly guiding planar waveguides even with a core layer of sub-micron thickness. Furthermore, domain engineering of LNOI was studied systematically for phase-matching nonlinear photonics. CMOScompatible domain engineering of LNOI is gradually accelerating the development of integrated nonlinear photonics, which will play an important role in integrated photonic quantum technology ${ }^{42}$. In addition, considering the difficulty of etching $\mathrm{LN}$, microstructure engineering using an easy-to-etch photonic material (e.g., SOI or silicon nitride) rib-loaded on LNOI is an attractive approach for hybrid integration of integrated photonic circuits, which not only obviates the necessity of etching LN but also combines the scalability of silicon photonics with the excellent electro-optic modulation of $\mathrm{LN}^{43}$.

Lithium niobate, as a traditional multifunctional material, has stimulated a photonics revolution as silicon did for electronics. Herein, we review the progress in microstructure and domain engineering of LNOI for integrated lithium niobate photonics, including photonic modulation and nonlinear photonics. Above all, ultra low-loss highly confined LN nanophotonic waveguides are the most fundamental building block ${ }^{17}$. Furthermore, the optical modulation device is at the heart of integrated lithium niobate photonic systems, as it encodes the RF signal. In Section "Microstructure engineering of LNOI for photonic modulation", the progress in the microstructure engineering of LNOI for the preparation of ultra low-loss highly confined nanophotonic waveguides and microphotonic modulators is described. Furthermore, we review the progress in on-chip nonlinear photonics involving highly confined photonic microstructures and phase-matching techniques for LNOI in Section "Microstructure engineering of LNOI for nonlinear integrated photonics". The focus is on the domain engineering of LNOI for QPM nonlinear photonics.

\section{Microstructure engineering of LNOI for photonic modulation}

Generally, the basic microstructure of integrated photonic circuits for photonic modulation contains ridge waveguides and microphotonic structures, including micro rings, microdisks, and microracetracks. 


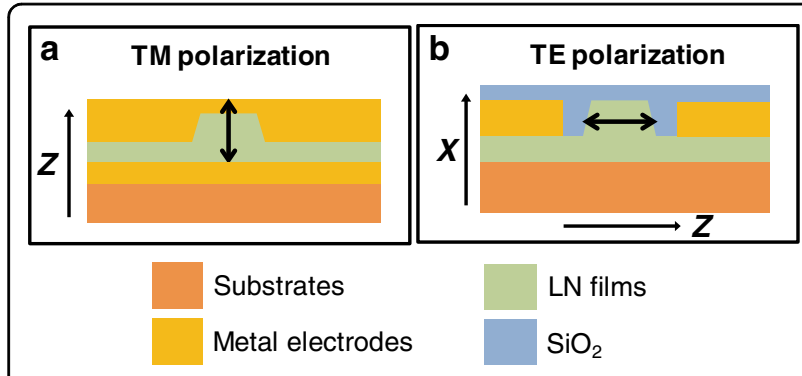

Fig. 3 Schematic of both TM $\mathbf{a}$ and TE $\mathbf{b}$ polarization of $L N$ nanophotonic waveguides on z-cut and x-cut LN films, respectively

Whispering-gallery-mode (WGM) microresonators are the most compact micro modulator with high confinement of light into small ring volumes, such as microrings, microdisks, and microracetracks ${ }^{44}$. Optical WGM microresonators have some exceptional properties, such as a small mode volume, a high power density, and a high-quality factor $Q(Q=\lambda / \Delta \lambda$, where $\lambda$ is the wavelength at which resonance occurs and $\Delta \lambda$ is the linewidth of the resonance wavelength). The refractive index changes when an electric field is applied to LN microresonators based on the electro-optic effect, which modifies the effective optical path length of the resonator. Thus, the resonance frequency of the microresonator shifts, thereby realizing electrical tuning.

In the early stage, efforts to construct microstructures on LNOI for photonic modulation were focused on lowloss nanophotonic waveguides and high-Q microresonators. Furthermore, MZI modulators integrated on LNOI platforms with advanced functionalities have been reported. In this section, both complementary microstructure engineering approaches for photonic circuits, i.e., direct etching of LNOI (monolithic integration) and using other photonic materials rib-loaded on LNOI (hybrid integration), are reviewed.

\section{Microstructure engineering by direct etching of LNOI Z-cut $L N$ films for TM polarization}

Generally, the development of WGM microresonators for integrated lithium niobate photonics is accompanied by the improvement of the LNOI preparation technique. In the early stage, $\mathrm{z}$-cut LN films were generally selected because of the limitations of lithographic techniques. Metal electrodes can be deposited between the substrate and the waveguide so that an electric field can be applied along the $\mathrm{z}$-axis to exploit the largest electro-optic coefficient, $r_{33}$, of LN, with light polarization along the $z$-axis (the electric field direction is mainly perpendicular to the LN film, which is called transverse magnetic (TM) polarization) (Fig. 3a).

To the best of our knowledge, a slab waveguide on LNOI was prepared for the first time by crystal ion slicing and wafer bonding (called the smart cut method) in $2004^{45}$. The electro-optic coefficients and refractive index of the LN film were found to be comparable to those of the bulk LN crystal. Ridge waveguides on LNOI were first fabricated by photolithography and $\mathrm{Ar}^{+}$beam milling ${ }^{46}$. However, the waveguide loss was not measured accurately and was estimated to be largely owing to the rough sidewalls caused by the etching process. It must be noted that the large mismatch in the thermal expansions of $\mathrm{LN}$ and $\mathrm{SiO}_{2} / \mathrm{Si}$ substrates can generate strong thermal stress during the bonding and layer transfer processes. Park et $\mathrm{al}^{47}$ introduced laser irradiation for the layer transfer process to promote localized layer exfoliation on an LN donor substrate and obtained a $6 \mathrm{~mm}^{2}$ LNOI sample with a microdisk structure. Later, Guarino et al. ${ }^{48}$ introduced benzocyclobutene $(\mathrm{BCB})$ as an adhesive layer between the metal electrode and LN film to reduce thermal stress and successfully prepared a centimeter-sized LNOI sample ${ }^{49}$. More importantly, an electro-optically tuneable WGM microresonator was introduced for the time on an LNOI platform. The transmission spectrum was tuned by the electro-optic effect, with a frequency tunability of $0.14 \mathrm{GHz} \mathrm{V}^{-1}$. However, BCB did not allow hightemperature annealing to repair the implantationinduced defects, which resulted in an electro-optical activity that was approximately $50 \%$ that of the bulk LN crystal and a large propagation loss of approximately $17 \mathrm{~dB} \mathrm{~cm}^{-1}$. Nevertheless, the study validated the viability of integrated lithium niobate photonics and stimulated further research on ultralow-loss sub-micron waveguides. $\mathrm{Hu}$ and co-workers prepared directly bonded LNOI without a BCB adhesive layer, which allowed hightemperature annealing to minimize ion-implantationinduced defects. Based on this, LN photonic wires with small cross-section dimensions of $1 \times 0.73 \mu \mathrm{m}^{2}$ were fabricated on an LNOI substrate (Fig. 4a) etched by inductively coupled plasma (ICP) $\mathrm{Ar}^{+}$milling ${ }^{50}$. The measured propagation loss was $9.9 \mathrm{~dB} \mathrm{~cm}^{-1}$ at a $1.55 \mu \mathrm{m}$ wavelength. Bo and co-workers fabricated LN microdisks with an undercut structure on an LNOI platform by UV photolithography, $\mathrm{Ar}^{+}$plasma etching, and HF etching ${ }^{51}$. The microdisk resonator coupled with a tapered fiber exhibited a quality factor of $1.19 \times 10^{6}$, which was two orders of magnitude higher than that of the reference microring resonator ${ }^{48}$.

\section{$X$-cut LN films for TE polarization}

However, z-cut LN films only work for TM polarization and are not suitable for on-chip integration. Therefore, $\mathrm{x}$ or y-cut LN films were adopted, with light polarization along the $z$-axis (the electric field direction is mainly parallel to the LN film, which is called transverse electric (TE) polarization) to exploit the electro-optic coefficient $r_{33}$. For this, metal electrodes must be deposited on both 


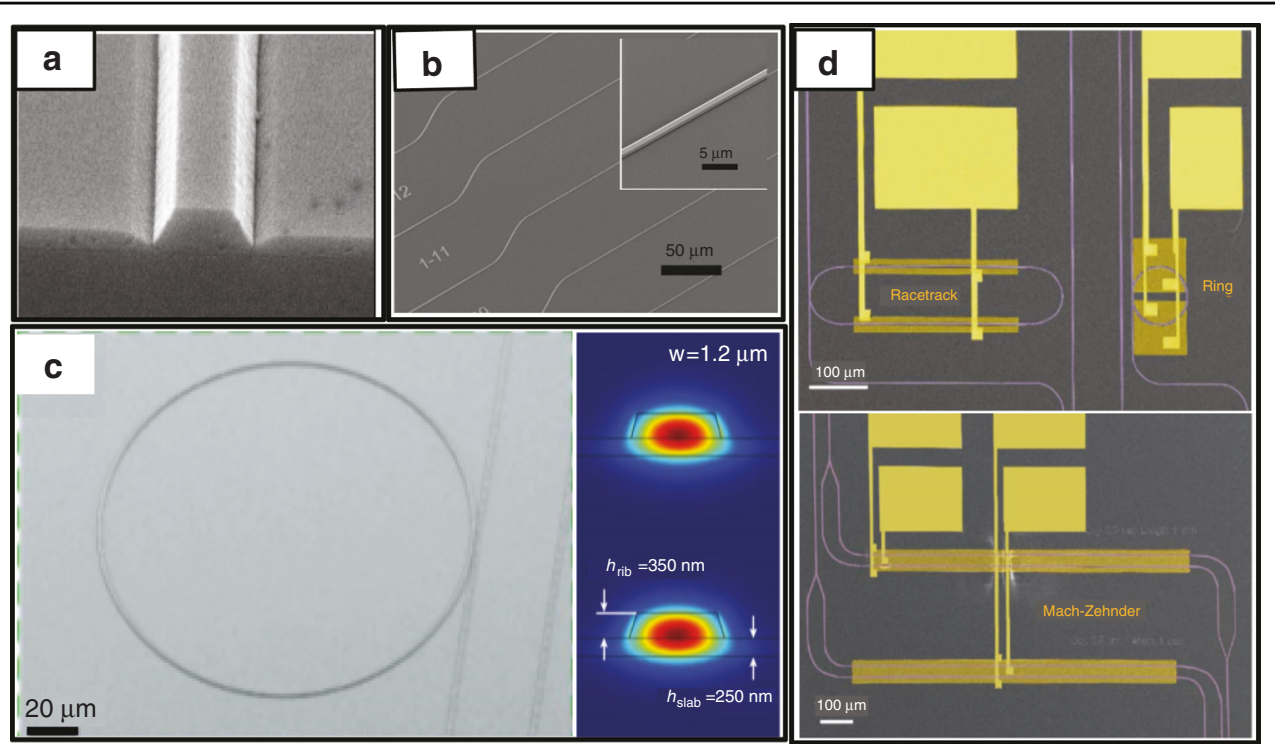

Fig. 4 a Image of microchannel ridge guides (photonic wires) with a $1 \mu \mathrm{m}$ top width ${ }^{50,154}$; Copyright 2009, Optical Society of America. b Array of LN waveguides with slightly different widths, with the inset showing a typical uniform LN waveguide with a fixed width ${ }^{53}$; Copyright 2017, Optical Society of America. c Image of a microring resonator consisting of dry-etched sub-wavelength waveguides with extracted propagation losses as low as $2.7 \mathrm{~dB} \mathrm{~m}^{-1}$ (left), as well as simulated optical modes in a bent (upper right) and straight (lower right) waveguides ${ }^{55}$; Copyright 2017, Optical Society of America. d Microphotonic devices including sub-micron waveguides, ring resonators, racetrack resonators, and MZI modulators integrated on an LNOI platform ${ }^{54}$; Copyright 2018, Optical Society of America

sidewalls of the waveguide channel, and an electric voltage should be applied along the z-axis of LN (Fig. 3b). As mentioned above, traditional LN modulators have weak optical confinement due to the low refractive index contrast. Therefore, metal electrodes are generally placed far from the waveguide to reduce the propagation loss due to metal absorption. Importantly, the much stronger optical confinement of channel waveguides in LNOI allows the electrodes to be placed close (micron-scale distance) to the sidewall of the waveguides, which results in a good overlap between the optical and microwave fields as well as strong phase modulation.

Bernal and co-workers improved the etching process to prepare a ridge waveguide with a smooth sidewall and reduced the propagation loss to $5 \mathrm{~dB} \mathrm{~cm}^{-1}$ on an $\mathrm{x}$-cut LNOI platform ${ }^{52}$. Wang and co-workers used an etched amorphous silicon (a-Si) mask as a resist to transfer the pattern to x-cut LN layers followed by $\mathrm{Ar}^{+}$milling. A uniform waveguide was prepared with a propagation loss of $3.0 \pm 0.2 \mathrm{~dB}$ $\mathrm{cm}^{-1} 53$. More excitedly, an array of LN waveguides with slightly different widths was successfully patterned on an LNOI platform with minimal surface roughness to obtain a manageable scattering loss, as shown in Fig. 4b. Later, microring resonators, racetrack resonators, and MZI modulators were integrated on the $\mathrm{x}$-cut LNOI platform by directly shaping the LN thin films into sub-wavelength waveguide channels (Fig. 4d) ${ }^{54}$. For racetrack resonators, the electric fields applied to the two racetrack arms were in the same direction to double the phase shifts in the two arms.
The electro-optic efficiency was measured as $7.0 \mathrm{pm} \mathrm{V}^{-1}$, which was similar to that of the ring resonator. For the MZI modulator, the half-wave voltage was $9 \mathrm{~V}$ with $2 \mathrm{~mm}$-long electrodes. Thus, the product of the switching voltage and electrode length was $1.8 \mathrm{~V} \mathrm{~cm}$, which is nearly an order of magnitude lower than that of traditional MZI modulators ${ }^{7}$. However, the propagation loss of the sub-wavelength waveguides of $3 \mathrm{~dB} \mathrm{~cm}^{-1}$ limits the application of the LNOI photonic platform, which arises because of the sidewall roughness caused by the etch mask.

By optimizing the ICP reactive-ion etching process for an LNOI platform, Zhang and co-workers fabricated subwavelength waveguides with a width of $1.2 \mu \mathrm{m}$ and a low propagation loss of $2.7 \mathrm{~dB} \mathrm{~m}^{-1}$. Furthermore, they demonstrated a nearly critically coupled microring resonator with an ultrahigh $Q$ of $5 \times 10^{6}$ (Fig. $\left.4 \mathrm{~b}\right)^{55}$. This important finding expanded the applicability of the LNOI photonic platform. Using a similar etching process, an integrated MZI modulator with a half-wave voltage of $1.4 \mathrm{~V}$ was fabricated on an LNOI platform, which is compatible with CMOS drive voltages ${ }^{56}$. Furthermore, several MZI modulators with various microwave signal line widths and device lengths were successfully integrated on an LNOI platform. The integrated MZI modulator with a small size of several millimeters could overcome the voltagebandwidth trade-off ${ }^{57}$, exhibiting very high bandwidths of up to $100 \mathrm{GHz}$. Moreover, an integrated MZI modulator on an LNOI platform demonstrated an unprecedented high electro-optic response of up to $500 \mathrm{GHz}^{58}$. 


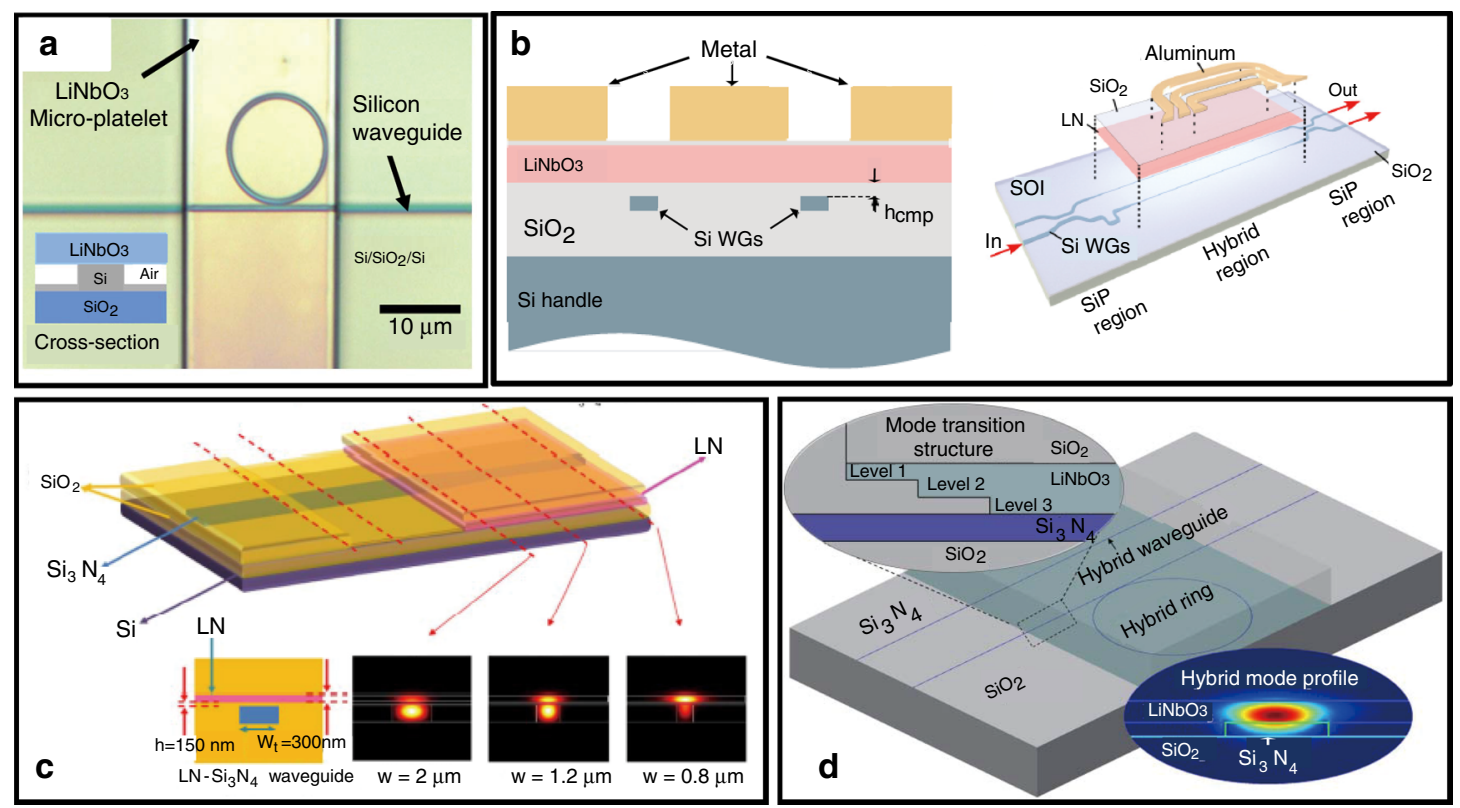

Fig. 5 a Optical micrograph of a hybrid $\mathrm{Si}-\mathrm{LiNbO}_{3}$ microring resonator ${ }^{59}$; Copyright 2011, Optical Society of America. b Schematic of the hybrid $\mathrm{Si}-\mathrm{LiNbO}_{3} \mathrm{MZI}$ modulator showing its cross-section ${ }^{63}$; Copyright 2018, Optical Society of America. c Schematic of the structure and cross-sections of hybrid $\mathrm{Si}_{3} \mathrm{~N}_{4}-\mathrm{LiNbO}_{3}$ waveguides, and simulated profiles of the fundamental TE modes at $1540 \mathrm{~nm}^{65}$; Copyright 2017, Optical Society of America. d Schematic of hybrid $\mathrm{Si}_{3} \mathrm{~N}_{4}-\mathrm{LiNbO}_{3}$ waveguides with a terrace shape ${ }^{66}$; Copyright 2018, Optical Society of America

In summary, the smart cut method for the preparation of wafer-scale LNOI samples (NanoLN, Jinan Jingzheng Electronics Co., Ltd.) greatly promoted the development of integrated lithium niobate photonics. On the one hand, the direct bonding between LN films and substrates without an adhesive layer allowed high-temperature annealing of the LNOI sample to minimize ionimplantation-induced defects. Then, the propagation loss of the nanophotonic LN waveguide was progressively reduced by optimizing the ICP reactive-ion etching process. On the other hand, the substitution of a z-cut LN film by an $x$-cut LN film greatly promoted the integration level of photonic circuits. The channel patterns with electrodes were easily transferred to an $\mathrm{x}$-cut LN film, while the bottom electrode generally occupied the whole plane for a z-cut LN film. Importantly, the gold electrodes can be placed very close to the edge of a resonator on an $\mathrm{x}$-cut LN film, which results in strong phase modulation without affecting the $\mathrm{Q}$ factor.

\section{Microstructure engineering using other photonic materials rib-loaded on LNOI}

As mentioned above, no existing photonic platform can deliver an optimal overall MWP system performance. There are strict requirements for the laboratory equipment for the direct etching of LN. Thus, microstructure engineering for integrated photonic circuits is often carried out using other photonic materials rib-loaded on LNOI.
Generally, this involves two-hybrid approaches: LN thin films are bonded onto silicon photonic circuits as a top cladding, and other photonic materials are rib-loaded on LNOI as a waveguide core. The electro-optic modulation under hybrid integration results from the overlap of the evanescent tail of the guided mode in the $\mathrm{LN}$ region.

\section{LN films as top cladding}

In 2011, Lee et al. ${ }^{59}$ demonstrated hybrid $\mathrm{Si}-\mathrm{LiNbO}_{3}$ electro-optically tunable ring resonators with free-standing z-cut $\mathrm{LN}$ thin films directly bonded to a $\mathrm{Si}$ microring resonator (Fig. 5a). The free-standing $\mathrm{LN}$ thin film was bonded onto the Si microring resonator as a top cladding, and an effective electro-optic coefficient of $1.7 \mathrm{pm} \mathrm{V}^{-1}$ was obtained for the TE mode, which is approximately one-fifth $\gamma_{13}\left(8.6 \mathrm{pm} \mathrm{V}^{-1}\right)$. Unfortunately, the largest electro-optic coefficient, $\gamma_{33}$, was not exploited. Chen and Reano ${ }^{60}$ used $\mathrm{BCB}$ as an intermediate bonding layer to fabricate a hybrid $\mathrm{Si}-\mathrm{LiNbO}_{3}$ microring resonator. Because of the absence of an integrated electrode, the voltage applied at both ends of the device was much larger, as the $\mathrm{SiO}_{2}$ layer consumed most of the voltage. Later, a hybrid $\mathrm{Si}-\mathrm{LiNbO}_{3}$ microring resonator (z-cut LN thin film) with an integrated electrode was reported, which offered low-voltage tunability ${ }^{61}$. The microresonator showed a quality factor of 11,500 and exhibited a resonance tuning of $12.5 \mathrm{pm} \mathrm{V}^{-1}$ for the TM mode, which used the largest electro-optic coefficient of $\mathrm{LN}, \gamma_{33}$. Because of the limitation of the annealing 
temperature due to $\mathrm{BCB}$, a pre-annealing process was employed to repair the crystal lattice and improve the electro-optic properties of the LN film. Here, the LN thin film was transferred onto a $\mathrm{Si}$ wafer and annealed at $1000^{\circ} \mathrm{C}$ before bonding to the Si microring resonators. Thus, an electro-optic microresonator on a hybrid $\mathrm{Si}-\mathrm{LiNbO}_{3}$ platform with a bandwidth of up to $5 \mathrm{GHz}$ was demonstrated for the first time ${ }^{62}$.

However, the refractive index of $\mathrm{Si}(\sim 3.4)$ is much higher than that of LN ( 2.2), which dramatically decreases the confinement factor in the $\mathrm{LN}$ region. It was found by simulation that the fraction of the optical mode power in $\mathrm{LiNbO}_{3}$ is only $11 \%$ for the TE mode. Weigel et al. ${ }^{63}$ introduced a thin $\mathrm{SiO}_{2}$ layer $(150 \mathrm{~nm})$ between an $\mathrm{x}$-cut LN thin film and a Si waveguide. As shown in the cross-section schematic of a hybrid MZI modulator (Fig. 5b), the LN thin film was oxide-bonded to the patterned and planarized silicon photonic circuits with a $\mathrm{SiO}_{2}$ interlayer. It was found that the thickness of the $\mathrm{SiO}_{2}$ layer has a significant impact on the bandwidth and $V_{\pi} L$ of MZI modulators. For this configuration, the fraction of the optical mode power in the $\mathrm{LN}$ region increased to $81 \%$. The hybrid $\mathrm{Si}-\mathrm{LiNbO}_{3} \mathrm{MZI}$ modulator with a length of $0.5 \mathrm{~cm}$ achieved a $3 \mathrm{~dB}$ electrical modulation bandwidth of $106 \mathrm{GHz}$ and a $V_{\pi} L$ of $6.7 \mathrm{~V} \mathrm{~cm}$.

Compared with $\mathrm{Si}, \mathrm{Si}_{3} \mathrm{~N}_{4}$ has a slightly smaller refractive index ( 1.98), a lower material loss, and a broader transparency window ${ }^{64}$. Chang et al. ${ }^{65}$ designed a hybrid $\mathrm{Si}_{3} \mathrm{~N}_{4}-\mathrm{LN}$ waveguide by bonding $\mathrm{LN}$ thin films onto a $\mathrm{Si}_{3} \mathrm{~N}_{4}$ waveguide layer on a Si substrate. As shown in Fig. 5c, it was found by simulation that the confinement factors of the two cores are sensitive to the $\mathrm{Si}_{3} \mathrm{~N}_{4}$ ridge width, which is attributed to the approximate refractive index contrast between $\mathrm{Si}_{3} \mathrm{~N}_{4}$ and LN. This configuration may be promising for a wide range of chip-level photonic applications.

However, the direct bonding of $\mathrm{LN}$ onto a predefined $\mathrm{Si}_{3} \mathrm{~N}_{4}$ or $\mathrm{Si}$ waveguide resulted in a significant mode transition loss at the interface due to the substantial disparity in effective indices and mode profiles. Thus, a mode converter structure must be designed to achieve a low transition loss from the waveguide cores to the hybrid core-cladding waveguide region. As shown in Fig. 5d, an LN film with a terrace shape was etched and then bonded onto the $\mathrm{Si}_{3} \mathrm{~N}_{4}$ waveguide on an $\mathrm{SOI}$ substrate. It was found by simulation that the transition loss was reduced from 2.67 to $0.81 \mathrm{~dB}$ at the interface ${ }^{66}$.

Furthermore, Cai and co-workers designed vertical adiabatic couplers (VACs), which transferred the optical powerfully, rather than partially, between the two layers ${ }^{43}$. For this configuration, a nanophotonic $\mathrm{LN}$ waveguide was designed on the top LN film, serving as a phase modulator, as shown in the cross-section schematic of the hybrid $\mathrm{Si}-\mathrm{LiNbO}_{3}$ waveguide. The interfaces between the bottom silicon inverse tapers and top superimposed LN waveguides couple light up and down between the two layers, which are named VACs. After optimizing the parameters of the hybrid MZI modulator, it exhibited an insertion loss of $2.5 \mathrm{~dB}$, a $V_{\pi} L$ of $2.2 \mathrm{~V} \mathrm{~cm}$, an electro-optic bandwidth of at least $70 \mathrm{GHz}$, and modulation rates up to $112 \mathrm{Gbit} \mathrm{s}^{-1}$. Therefore, it had significantly increased modulation efficiency compared with the directly bonded waveguide.

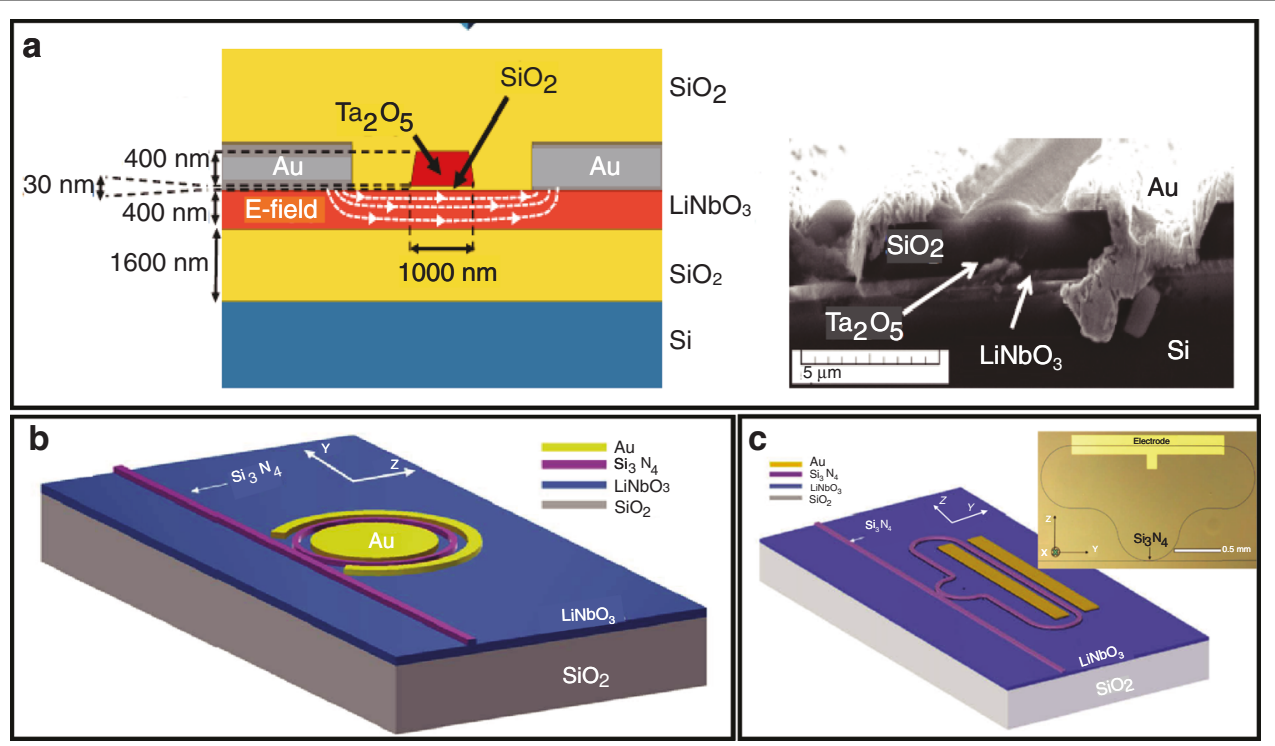

Fig. 6 a Cross-section of the hybrid $\mathrm{Ta}_{2} \mathrm{O}_{5}-\mathrm{LNOI}$ waveguide ${ }^{67}$; Copyright 2013, Optical Society of America. b Schematic of the tuneable hybrid $\mathrm{Si}_{3} \mathrm{~N}_{4}-\mathrm{LNOI}$ microring resonator ${ }^{72}$; Copyright 2019, Optical Society of America. c Schematic and microscope image of a tunable hybrid $\mathrm{Si}_{3} \mathrm{~N}_{4}-\mathrm{LNOI}$ racetrack resonator ${ }^{73}$. Copyright 2019, Optical Society of America 


\section{LNOI as a substrate}

Considering the difficulties in the direct etching of LN, LNOI can be used as a substrate with a rib-loaded material having a refractive index that forms a hybrid waveguide. Rabiei et al. ${ }^{67}$ designed a single-mode submicron ridge composite waveguide with a tantalum pentoxide $\left(\mathrm{Ta}_{2} \mathrm{O}_{5}\right)$ rib region loaded onto an LNOI platform (Fig. 6a). The $\mathrm{Ta}_{2} \mathrm{O}_{5}$ rib region was prepared by selective oxidation of $\mathrm{Ta}^{68}$. Based on the composite waveguide, a microring resonator and an MZI modulator were prepared on a y-cut LNOI platform with a quality factor of approximately $7.2 \times 10^{4}$ and a $V_{\pi} L$ of $4 \mathrm{~V} \mathrm{~cm}$. Another index-matched material, $\mathrm{Ge}_{23} \mathrm{Sb}_{7} \mathrm{~S}_{70}$ chalcogenide, was also used to fabricate a composite waveguide, which simplified the fabrication process and reduced the propagation loss to $1.2 \mathrm{~dB} \mathrm{~cm}^{-1}{ }^{69}$. Nevertheless, $\mathrm{Ta}_{2} \mathrm{O}_{5}$ and $\mathrm{Ge}_{23} \mathrm{Sb}_{7} \mathrm{~S}_{70}$ chalcogenides are not conventional photonic materials and are incompatible with CMOS processing.

Hybrid Si-LN waveguides were fabricated by lithography on $\mathrm{Si}-\mathrm{LN}$ platforms ${ }^{70}$, exhibiting a propagation loss of $2.5 \mathrm{~dB} \mathrm{~cm}^{-1}$ in the mid-infrared range. The $V_{\pi} L$ of the integrated MZI modulator was high at $27 \mathrm{~V} \mathrm{~cm}$ because of the large gap between the electrodes and the waveguide. In addition, silicon nitride was used to fabricate a composite waveguide by rib deposition on $\mathrm{LNOI}^{71}$. First, the silicon nitride layer was deposited on an LN film by PECVD. Then, ridge optical waveguides were patterned by standard lithography. Rao et al. ${ }^{57}$ prepared compact MZI modulators with a hybrid SiN-LNOI composite waveguide using a $2 \mu \mathrm{m}$-thick BCB layer as the top cladding. The high-performance devices showed a $V_{\pi} L$ of $3.1 \mathrm{~V} \mathrm{~cm}$ under DC and of less than $6.5 \mathrm{~V} \mathrm{~cm}$ with a bandwidth of up to $50 \mathrm{GHz}$. Furthermore, a tunable hybrid $\mathrm{Si}_{3} \mathrm{~N}_{4}-\mathrm{LN}$ microring resonator (Fig. $\left.6 \mathrm{~b}\right)^{72}$ and a racetrack resonator (Fig. $6 \mathrm{c})^{73}$ were lithographically deposited on an $\mathrm{x}$-cut LNOI platform with a resonance tunability of up to 1.78 and $2.9 \mathrm{pm} \mathrm{V}^{-1}$, respectively. Both microresonators employed an air top cladding and exhibited a quality factor of $10^{5}$ for TE polarization.

In conclusion, the hybrid integration of photonic materials with an LN film is an effective approach for integrated photonics that can avoid the etching of LN. However, regardless of whether the LN film works as a top cladding or a substrate, the transition loss between the two layers is still a problem for this hybrid waveguide. Although the design of VACs nearly resolved the transition loss problem, the nanophotonic LN waveguide was still adopted, and the waveguide parameters must be precisely controlled for high modulation efficiency and low optical loss. This does not fully reflect the strengths of hybrid integration, which avoids the etching of LN. Therefore, the hybrid integration approach still needs further improvement.

\section{Microstructure engineering of LNOI for nonlinear integrated photonics}

High-efficiency, compact, and integration-compatible wavelength converters using optical waveguides involve nonlinear integrated photonics ${ }^{74-76}$. In nonlinear optics, the polarization intensity of the dielectric is related to the intensity of the incident light wave as $\mathrm{P}=$ $\varepsilon_{0}\left(\chi^{(1)} E+\chi^{(2)} E E+\chi^{(3)} E E E+\ldots\right)$. The first term represents the first-order linear component, while the nonlinear optics is related to the higher-order components represented typically by the second- and third-order nonlinear optical susceptibilities $\chi^{(2)}$ and $\chi^{(3)}$, respectively). The higher-order components are generally ignored. However, for nonlinear integrated photonics on semiconductor waveguides, third-order nonlinear optical interactions have been widely exploited because of some common photonic materials, such as $\mathrm{SiO}_{2}$ and $\mathrm{Si}$, lack $\chi^{(2)}$, the second-order nonlinear optical susceptibility ${ }^{75,77}$. In addition, second-order nonlinear optical processes were investigated for some photonic materials, such as GaAs and AlGaAs, as $\chi^{(2)}$ is typically stronger than $\chi^{(3)} 78$.

In the case of nonlinear integrated photonics on LNOI, second-order nonlinear frequency conversion has been widely researched because $\chi^{(2)}$ (linear electro-optic coefficient $r_{33}=3.09 \mathrm{pm} \mathrm{V}^{-1}$ ) is much larger than $\chi^{(3)}$ (the nonlinear refractive index is $\sim 10^{-15} \mathrm{~cm}^{2} \mathrm{~W}^{-1}$ at $1064 \mathrm{~nm}$ ) for $\mathrm{LN}^{79}$. Generally, photonic microstructures used for frequency conversion are designed based on strong mode confinement because the nonlinear effect can be significantly enhanced inside a small modal volume due to the increased field strength and temporal confinement of the interacting modes ${ }^{80}$. Thus, high-Q microresonators with light confined in a cavity for a long time serve as a promising avenue for nonlinear frequency conversion. Considering that the conversion efficiency of highconfinement resonators mainly depends on the modal overlap between the fundamental modes and higher harmonics ${ }^{81,82}$, strict phase matching is not required. However, obvious phase mismatch between the interacting waves often results from material dispersion. Therefore, several phase-matching techniques, as well as the associated waveguide microstructure engineering approaches, have been explored for second harmonic generation (SHG) processes, including modal phase matching (MPM), mode-shape modulation, and domain engineering $^{78}$.

\section{Microstructure engineering of LNOI for phase-matching- free nonlinear optics Microring cavities for frequency conversion}

WGM microresonators as optical microcavities have been exploited for many different applications, including lasing on a chip, electro-modulation, nonlinear frequency conversion, and frequency comb generation ${ }^{83-85}$. Wang 
et al. $^{86}$ demonstrated an on-chip integrated nonlinear frequency conversion process using microdisk resonators on an LNOI platform. The microdisk was undercut by wet etching using HF followed by electron beam lithography patterning and $\mathrm{Ar}^{+}$milling. With the aid of a silica fiber to couple light into and out of the microresonators, on-chip SHG was obtained with a conversion efficiency of $0.109 \mathrm{~W}^{-1}$. In particular, spontaneous parametric downconversion was achieved using microdisk resonators over a bandwidth of $400 \mathrm{~nm}^{87}$. A quasi-TM visible pump photon at a wavelength of $774.66 \mathrm{~nm}$ produced a pair of $1549.32 \mathrm{~nm}$ photons with orthogonal quasi-TM and quasi-TE polarizations, which is the operating principle of an entangled source. Nevertheless, microresonators with specific structures need to be designed to achieve highly efficient nonlinear frequency conversion ${ }^{80}$. Moreover, cascaded Raman scattering and frequency-doubled emission of Raman lines were observed at the microring resonators with a $Q$ value of up to $10^{688,89}$. An innovative tuneable coupling scheme to optimize the coupling was demonstrated for nonlinear optical processes by changing the distance between the coupling waveguide and the WGR in the $\mathrm{z}$-direction.

\section{Microring cavities have been used to realize optical frequency combs}

Microring cavities have been used to realize optical frequency combs ${ }^{90-92}$, whose spectra consist of numerous discrete, equidistant laser lines, which was suggested to revolutionize wavelength division multiplexing in optical telecommunication. The currently available optical frequency combs are based on femtosecond lasers ${ }^{93}$. Nevertheless, further increasing the repetition rate into the frequency range above $10 \mathrm{GHz}$ was an attractive challenge $^{94}$. Recently, combs with wide spectra have been generated by the third-order Kerr nonlinearity $\left(\chi^{(3)}\right)$ from a monolithic microresonator in some photonic materials $^{95-97}$. However, the electro-optic modulator for tuneable filtering cannot be monolithically integrated into a single chip because of the lack of $\chi^{(2)}$. Wang et al. ${ }^{98}$ demonstrated frequency comb generation $\left(\chi^{(3)}\right.$ microring resonator) and electro-optic manipulation (filtering and electro-optic modulation via a $X^{(2)}$ resonator) on a single integrated LNOI chip. The monolithic LNOI photonic circuits were patterned by directly shaping the LN thin films into a sub-wavelength waveguide channel by a similar standard process. A TE-polarized comb was generated from 1400 to $2100 \mathrm{~nm}$ with a line spacing of approximately $250 \mathrm{GHz}$. Meanwhile, different target comb lines can be selected by the $X^{(2)}$ microring resonator by applying different bias voltages. However, strong phase noise and stability are the most significant problems for Kerr frequency combs because of the complex nature of the third-order Kerr effect.
An electro-optic modulation is an alternative approach for producing optical frequency combs in a resonator with excellent stability and controllability ${ }^{99}$. This principle exploits the second-order nonlinearity involving a microwave field that modulates the optical wave within the nonlinear crystal. The resonance frequencies in the optical resonator are separated by the free spectral range (FSR), which must be designed to match the frequency of the microwave field, enabling sum- and differencefrequency generation of sidebands ${ }^{100}$. Rueda et al. ${ }^{101}$ demonstrated electro-optic comb generation based on an LN WGM resonator embedded in a copper microwave cavity. The WGM resonator was a convex-shaped disk with a radius of $2.45 \mathrm{~mm}$ and a thickness of $0.4 \mathrm{~mm}$, resulting in an FSR of approximately $8.9 \mathrm{GHz}$ at a pump wavelength of $1549 \mathrm{~nm}$. With pump light of $320 \mu \mathrm{W}$ and a low-power microwave modulation field of $20 \mathrm{~dB}$ at $8.9 \mathrm{GHz}$ coupled into the WGM resonator, the generated comb spanned $11 \mathrm{~nm}$ with 180 lines in the C-band. It was demonstrated theoretically that strong phase modulation and high $\mathrm{Q}$ are crucial for microresonators to generate flat and broad electro-optic frequency combs ${ }^{102}$. Zhang et al. ${ }^{102}$ realized a broader electro-optic comb generator using high-Q microracetrack resonators integrated on a single LNOI photonic platform. The broad electro-optic frequency comb spanned $80 \mathrm{~nm}$ over part of the telecommunication C-band, the entire L-band, and part of the U-band with over 900 lines spaced by $10.453 \mathrm{GHz}$, which was generated by the resonator with a size of $200 \mu \mathrm{m} \times 6.2 \mathrm{~mm}$ modulated by a microwave field at a frequency near the resonator FSR of $10 \mathrm{GHz}$.

\section{Metasurfaces}

Metasurfaces consisting of nanoantennas are often used to realize enhanced optical nonlinearities ${ }^{103-106}$, as nonlinear plasmonic effects can arise from the coherent oscillations of conduction electrons near the surface of the metal structures ${ }^{81}$. A nanophotonic LN waveguide patterned with gradient metasurfaces was fabricated to achieve a monotonic increase in SHG power ${ }^{107}$. As shown in Fig. 7a, a gradient metasurface consisting of a number of identical phased antenna arrays was patterned along the top surface of the LN waveguide. Each array consisted of 35 amorphous silicon nanoantennas with a range of lengths. Generally, once optical power couples from the fundamental mode at the pump frequency, $\operatorname{TE}_{00}(\omega)$, to the fundamental mode at the $\mathrm{SH}$ frequency, $\mathrm{TE}_{00}(2 \omega)$, it immediately starts to be converted into higher-order waveguide modes at the $\mathrm{SH}$ frequency, $\operatorname{TE}_{\mathrm{mn}}(2 \omega)$ and $\mathrm{TM}_{\mathrm{mn}}(2 \omega)$, by the gradient metasurface. Theoretically, the unidirectional wavevector provided by the gradient metasurface prevents optical power coupling from $\mathrm{TE}_{\mathrm{mn}}(2 \omega)$ and $\mathrm{TM}_{\mathrm{mn}}(2 \omega)$ modes back to the $\mathrm{TE}_{00}(2 \omega)$ mode. Furthermore, the optical power carried by 

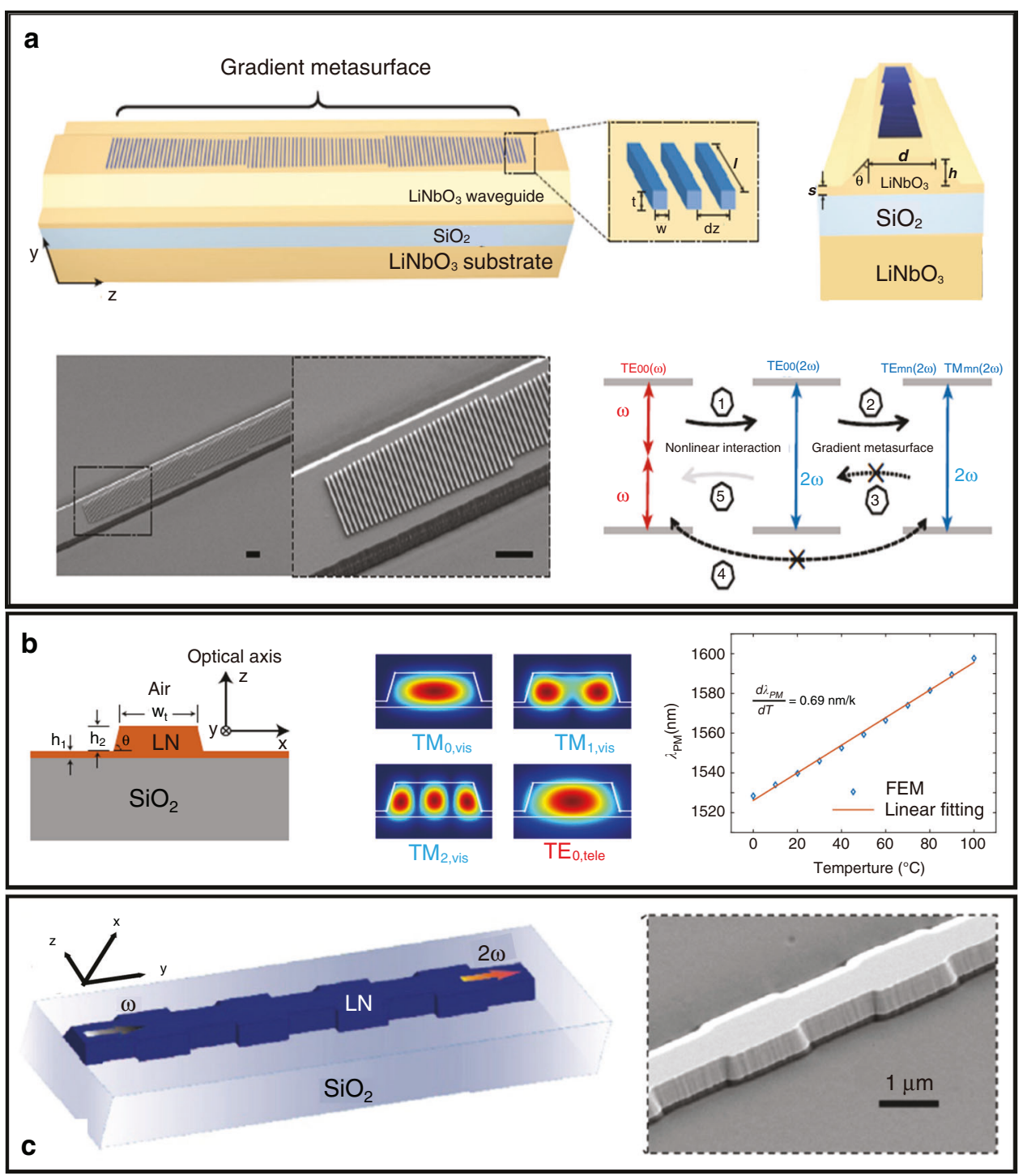

Fig. 7 a Schematic and working principle of the metasurface of a LN nanophotonic waveguide ${ }^{107}$; Copyright 2017, Springer Nature. b Schematic and simulated mode profiles of a nanophotonic waveguide and the modal-phase-matching condition ${ }^{111}$; Copyright 2018, Optical Society of America. c Schematic and SEM image of the periodically grooved structure of a nanophotonic waveguide ${ }^{53}$; Copyright 2017, Optical Society of America

$\mathrm{TE}_{\mathrm{mn}}(2 \omega)$ and $\mathrm{TM}_{\mathrm{mn}}(2 \omega)$ modes cannot be coupled back to the $\mathrm{TE}_{00}(\omega)$ mode yet. Therefore, the gradient metasurfaces break the symmetry of the coupling between the pump and SH signals, resulting in high-efficiency SHG of approximately $1000 \% \mathrm{~W}^{-1} \mathrm{~cm}^{-2}$, which is three orders of magnitude more efficient than the bare $\mathrm{LN}$ waveguide.

\section{Microstructure engineering of $\mathrm{LNOI}$ for phase-matching nonlinear optics}

In the most generic second-order nonlinear optical process, which is taken as an example here, three-wave mixing must satisfy energy conservation and momentum conservation.

$$
\omega_{3}=\omega_{1}+\omega_{2} \text { and } k_{3}=k_{1}+k_{2}
$$

where $k_{i}=n_{i} \omega_{i} / c$

Thus, momentum conservation becomes

$$
n_{3} \omega_{3}=n_{1} \omega_{1}+n_{2} \omega_{2}
$$

The three waves travel at different velocities due to the dispersion of the medium, corresponding to the different 
refractive indices $n_{1}, n_{2}$, and $n_{3}$. As a result, the refractive indices for the three waves need to be precisely controlled for phase matching.

\section{Modal phase matching}

MPM is a simple technique for phase velocity synchronism in nonlinear frequency conversion processes ${ }^{108}$. Because longer wavelengths have lower effective indices and the higher-order mode of the wavelengths has a lower effective index in a multimode waveguide, the fundamental modes of one or two of the longer wavelengths are phase-matched with a higher-order mode of shorter wavelength ${ }^{78}$. Generally, the type- 0 configuration is employed to achieve a high conversion efficiency. Wang and co-workers demonstrated the dependence of the effective mode indices $\left(n_{\text {eff }}\right)$ of both the fundamental mode at the incident wavelength and higher-order modes at the $\mathrm{SH}$ wavelength on the top width $\left(w_{\mathrm{t}}\right)$ of the LN waveguide with fixed initial thickness and sidewall angle. To exploit the large nonlinear coefficient $d_{33}$, phase matching was achieved between the first-order TE modes at the fundamental wavelength and the third-order $\mathrm{TE}$ modes at the $\mathrm{SH}$ wavelength, with normalized conversion efficiencies as high as $41 \% \mathrm{~W}^{-1} \mathrm{~cm}^{-2}$ at $w_{\mathrm{t}}=630 \mathrm{~nm}^{53}$. In addition, MPM was demonstrated between the fundamental TM mode at $1550 \mathrm{~nm}$ and the second-order TM modes at the $\mathrm{SH}$ wavelength on monolithic z-cut LNOI by changing the top width of the LN waveguide to approximately $590 \mathrm{~nm}^{109}$. SHG of the type-I configuration was also found for a nanophotonic LN waveguide between the fundamental quasi-transverse-electric mode $\left(\mathrm{TE}_{00}\right)$ and the higher-order quasi-transverse-magnetic $\mathrm{SH}$ mode $\left(\mathrm{TM}_{\mathrm{mn}}\right)$. This waveguide inevitably exhibited a low conversion efficiency due to the significantly weaker nonlinearity $\left(d_{31}\right)$ than in the type- 0 configuration $\left(d_{33}\right)$. On a sub-micron LN waveguide with a cross-section of $1.2 \mu \mathrm{W} \times 0.53 \mu \mathrm{W}$, the phase-matched SHG process occurs between the fundamental mode $\left(\mathrm{TE}_{00}\right)$ and the second-order $\mathrm{SH}$ mode $\left(\mathrm{TM}_{20}\right)$ at a wavelength of $1413 \mathrm{~nm}^{110}$. This SHG process generates an SH power of approximately $305 \mathrm{pW}$ with an incident power of $737 \mu \mathrm{W}$, achieving a normalized SHG efficiency of approximately $6.9 \% \mathrm{~W}^{-1} \mathrm{~cm}^{-2}$.

Even so, the type-I configuration was often employed for wavelength tunability of SHG because LN exhibits a significant thermo-optic birefringence with a value of approximately $4 \times 10^{-5} \mathrm{~K}^{-1}$ at room temperature. Thus, a temperature change of the device would result in a considerable variation in the material birefringence, which in turn shifts the phase-matched wavelength of the type-I SHG process. Based on the thermo-optic birefringence of $\mathrm{LN}$, highly tuneable efficient SHG with a tuning slope of $0.84 \mathrm{~nm} \mathrm{~K}^{-1}$ was demonstrated in a $\mathrm{z}$-cut $\mathrm{LN}$ nanophotonic waveguide by phase-matching the fundamental
quasi-TE mode in the telecom band with the third-order quasi-TM mode at the $\mathrm{SH}$ wavelength (Fig. $7 \mathrm{~b})^{111}$. The waveguide exhibited a theoretical normalized conversion efficiency of $22.2 \% \mathrm{~W}^{-1} \mathrm{~cm}^{-2}$ and an experimentally determined SHG efficiency of $4.7 \% \mathrm{~W}^{-1}$ for type-I intermodal phase matching.

\section{Mode-shape modulation}

is an alternative poling-free implementation for integrated QPM with periodic gratings ${ }^{112}$. Waveguides with periodically grooved structures were also fabricated on an $\mathrm{x}$-cut LNOI platform, which was adopted to realize phasematched SHG (Fig. 7c) ${ }^{53}$. The periodic perturbation in the periodically grooved waveguide generates space harmonics with new propagation constants to compensate for the phase mismatch ${ }^{113}$. Moreover, a hybrid $\mathrm{SiN}_{x}$-LNOI waveguide in which the width of the $\mathrm{SiN}$ rib was periodically modulated was fabricated to achieve QPM $\mathrm{SHG}^{114}$.

\section{Domain engineering of LNOI for phase-matching nonlinear optics}

In QPM theory, the phase variation is $\Delta k_{Q}=$ $k_{3}-k_{2}-k_{1}-k_{\Lambda}$, where the grating vector is $k_{\Lambda}=2 \pi / \Lambda$ for grating period $\Lambda$ analogous to wave vectors. The nonlinear coefficient is modulated with a period twice the coherence length $\left(l_{c}\right)$ of the interaction to offset the accumulated phase mismatch. The grating period is determined from the relevant radiation frequency and the working temperature according to the equation $\Lambda=2 \mathrm{l}_{c}=\frac{2 \pi}{k_{3}-k_{2}-k_{1}}$. Owing to the advantage of LNOI in integrated photonics, domain engineering of LNOI has attracted much interest for integrated frequencyconversion devices. The recently developed integrated frequency-conversion devices on PPLN films also involve hybrid and monoclinic approaches. For the hybrid approach, another photonic material was integrated on the PPLN film to guide the light, while frequency conversion was realized in the PPLN region. For the monoclinic approach, the ridge waveguide was etched in the PPLN region to form nanophotonic PPLN waveguides.

\section{PPLN waveguides on LNOI platforms}

PPLN waveguides on LNOI platforms are crucial for realizing highly efficient, compact, and integrationcompatible wavelength converters. Generally, PPLN is prepared by applying an external electric field using periodic electrodes. Gainutdinov and co-workers realized periodic poling of z-cut LN thin films on insulators using an atomic force microscope $\mathrm{e}^{115}$, which is favorable only for the TM polarization mode to exploit the large nonlinear coefficient $d_{33}$. Hence, the $\mathrm{x}$ - or $\mathrm{y}$-cut geometry is preferred because of the possibility of ease of processing on top of the thin film sample. Mackwitz et al. ${ }^{116}$ first 
fabricated periodically poled domain patterns in $\mathrm{x}$-cut LNOI. Rao et al. ${ }^{117}$ deposited poling electrodes on the same surface containing LN by filling the spaces of the pre-etched LN thin film, which can reduce the consumption of the electric field component parallel to the $x$-axis. In the early stages, the approach of hybrid integration was adopted for photonic materials rib-deposited onto PPLN films. Hybrid $\mathrm{SiN}_{x}-$ PPLN waveguides were formed by rib-loaded channels of $\mathrm{SiN}_{x}$ in the periodic domain region of $\mathrm{LN}$ thin films, with a final deposition of a $\mathrm{SiO}_{2}$ top cladding, as shown in Fig. 8a. It was found by simulation that the $\mathrm{SiN}_{x}$ ribs with a cross-section of $2000 \mathrm{~nm} \times 400 \mathrm{~nm}$ resulted in a poling period of approximately $5 \mu \mathrm{m}$ for TE-polarized pump light at $1580 \mathrm{~nm}$ phase matched with the $\mathrm{SH}$ wavelength. Chang et al. ${ }^{118}$ also achieved QPM SHG with an output power of $80 \mathrm{nW}$ at a $1530 \mathrm{~nm}$ pump power of $0.5 \mathrm{~mW}$ using hybrid $\mathrm{SiN}_{x}-\mathrm{PPLN}$ waveguides. The SHG exhibited a peaknormalized efficiency of $160 \% \mathrm{~W}^{-1} \mathrm{~cm}^{-2}$. The duty ratio of the periodically poled domain patterns in the $\mathrm{x}$-cut $\mathrm{LN}$ thin film still needs to be improved ${ }^{119}$. Although the hybrid waveguide obviated the necessity of LN etching, it was difficult to enhance the nonlinear conversion efficiency. Monolithic nanophotonic PPLN waveguides propagating along the $y$-axis were successfully prepared by standard lithography on a periodically poled x-cut $\mathrm{Mg}$ doped LN film (Fig. 8b) ${ }^{120}$. The nanophotonic PPLN waveguides with a cross-section of $1400 \mathrm{~nm} \times 600 \mathrm{~nm}$ exhibited a domain-inverted period of $\sim 4 \mathrm{~nm}$, which indicated that the fundamental TE modes at $1550 \mathrm{~nm}$ were phase matched with the fundamental TE modes at the SH wavelength. Moreover, SHG of $117 \mathrm{~mW}$ at $775 \mathrm{~nm}$ was achieved using a pump power of $220 \mathrm{~mW}$ with an absolute conversion efficiency of $53 \%$ in a $4 \mathrm{~mm}$-long device, corresponding to the normalized conversion efficiency of $2600 \% \mathrm{~W}^{-1} \mathrm{~cm}^{-2}$. With an optically monitored iterative poling, depoling, and repoling sequence to improve the domain-inverted structure of nanophotonic LN waveguides, conversion efficiency of up to $4600 \%$ $\mathrm{W}^{-1} \mathrm{~cm}^{-2}$ was achieved for SHG by pumping at approximately $1540 \mathrm{~nm}^{121}$.

\section{Periodically poled microresonators}

In addition, a PPLN thin film was exfoliated from a bulky PPLN wafer by the crystal ion slicing method ${ }^{122,123}$. It may have potential applications in nonlinear integrated photonics, such as micro rings and microdisks with periodic domain structures demonstrating QPM SHG ${ }^{124-127}$. A disk was fabricated by diamond polishing from a periodically poled LN wafer, which exhibited a maximum output power of $12.3 \mathrm{~mW}$ at a wavelength of $775 \mathrm{~nm}$, as observed by a frequency-doubling experiment. The pump power at the input of the cavity with a wavelength of $1.55 \mu \mathrm{m}$ was $25 \mathrm{~mW}$, with a maximum SHG conversion efficiency of approximately $0.5^{127}$. In addition, the z-cut LN microring can be periodically polled by an external electric field between the bottom aluminum plate electrode and the top radial nickel electrodes (Fig. 8c) ${ }^{128}$. The results for the periodically poled microring with a period of $7.46 \mu \mathrm{m}$ indicated that the fundamental TE mode was quasi-phase-matched with the SH TM mode to exploit the nonlinear coefficient $d_{31}$. By coupling with a pulley bus waveguide, the periodically poled microring resonator was pumped at approximately $1617 \mathrm{~nm}$, which yielded a QPM SHG conversion efficiency of up to $250,000 \% \mathrm{~W}^{-1}$ and absolute conversion efficiency of $15 \%$. The higher SHG efficiency is attributed to the stronger mode confinement of the microring due to the larger modal overlap as well as to the phase-matching due to the periodic domain structure.

In conclusion, LNOI is a promising platform for nonlinear integrated photonics. Generally, the phasematching condition is a critical factor in nonlinear frequency conversion processes. However, phase-matchingfree SHG has been achieved with high-efficiency conversion by a gradient metasurface on an on-chip integrated nonlinear photonic device. This finding will promote the development of nonlinear integrated photonics on LNOI. Of course, many integrated nonlinear photonic devices were fabricated by following phase-matching SHG, especially quasi-phase-matching. The preparation of PPLN films greatly promoted the nonlinear integrated photonics on LNOI, especially the nanophotonic PPLN waveguides, which realized a conversion efficiency of up to $4600 \%$ $\mathrm{W}^{-1} \mathrm{~cm}^{-2}$. In the future, nanophotonic PPLN waveguides will be applied in quantum technology to develop integrated quantum technology.

\section{Summary and prospects}

The recent progress in the microstructure and domain engineering of LNOI for integrated photonics was reviewed. It is feasible that microstructures can be constructed on LNOI platforms for photonic circuits, which are also compatible with CMOS technology. Ultralow-loss nanophotonic LN waveguides and high-Q WGM microresonators have been prepared on the LNOI platform, which enables the sophisticated manipulation of light signals. Compared with other photonic materials, $\mathrm{LiNbO}_{3}$ exhibits a high-performance electro-optic effect and nonlinear optical properties. On the one hand, the highbandwidth and low-drive-voltage electro-optical modulator is a critical component in optical fiber communication, which can achieve high-speed signal conversion between electricity and light waves with low power consumption. The integrated modulator on LNOI has been realized experimentally with a $100 \mathrm{GHz}$ bandwidth at a drive voltage of approximately $1 \mathrm{~V}$, while the conventional LN modulators consume high power with a drive voltage 

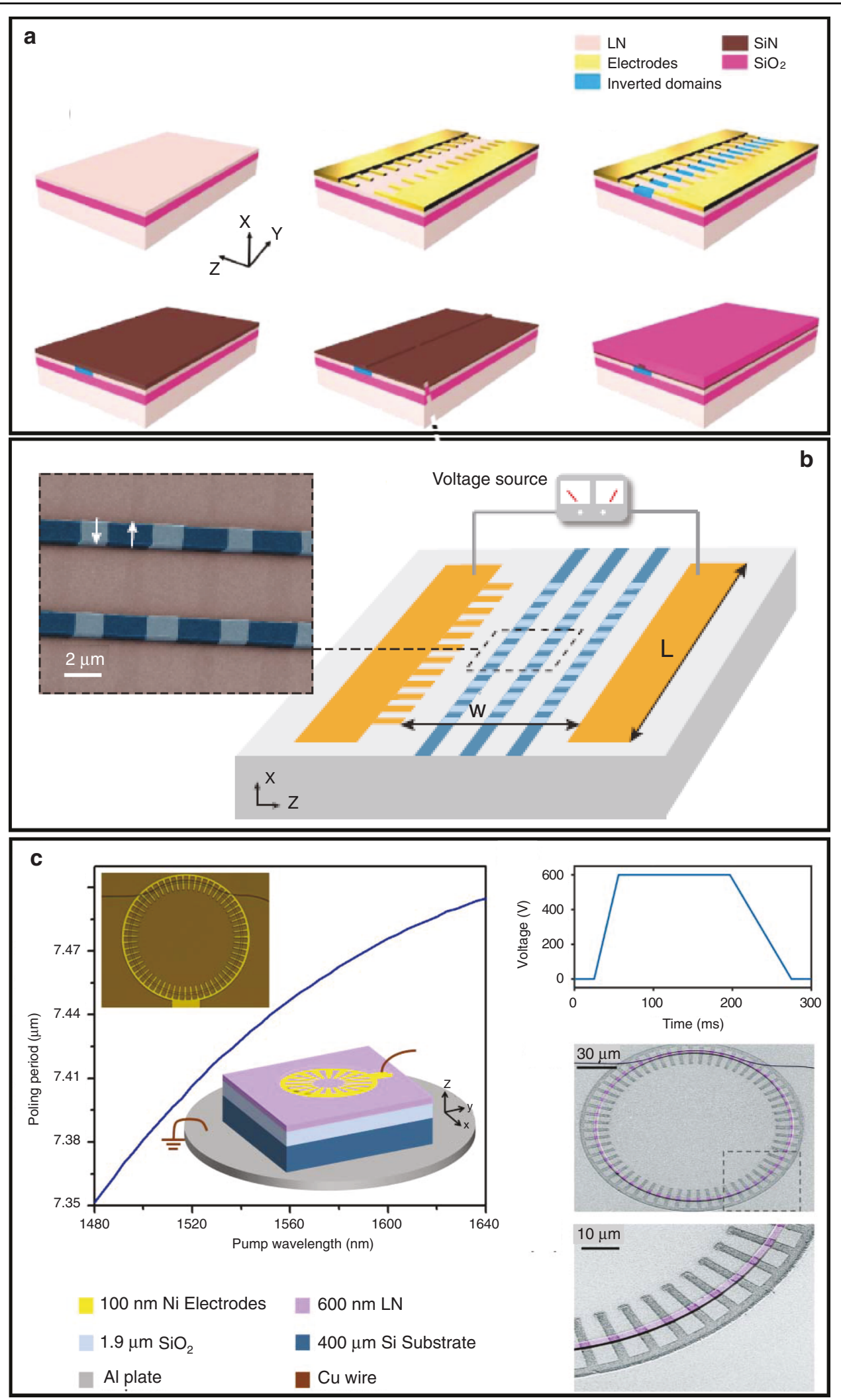

Fig. 8 a Schematic of the processing of a hybrid $\operatorname{SiN}_{x}-P P L N$ waveguide ${ }^{118}$; Copyright 2016, Optical Society of America. b Schematic and false-color SEM image of a periodically poled nanophotonic waveguide ${ }^{120}$; Copyright 2018, Optical Society of America. c Periodically poled LN microring resonator ${ }^{128}$; Copyright 2019, Optical Society of America 


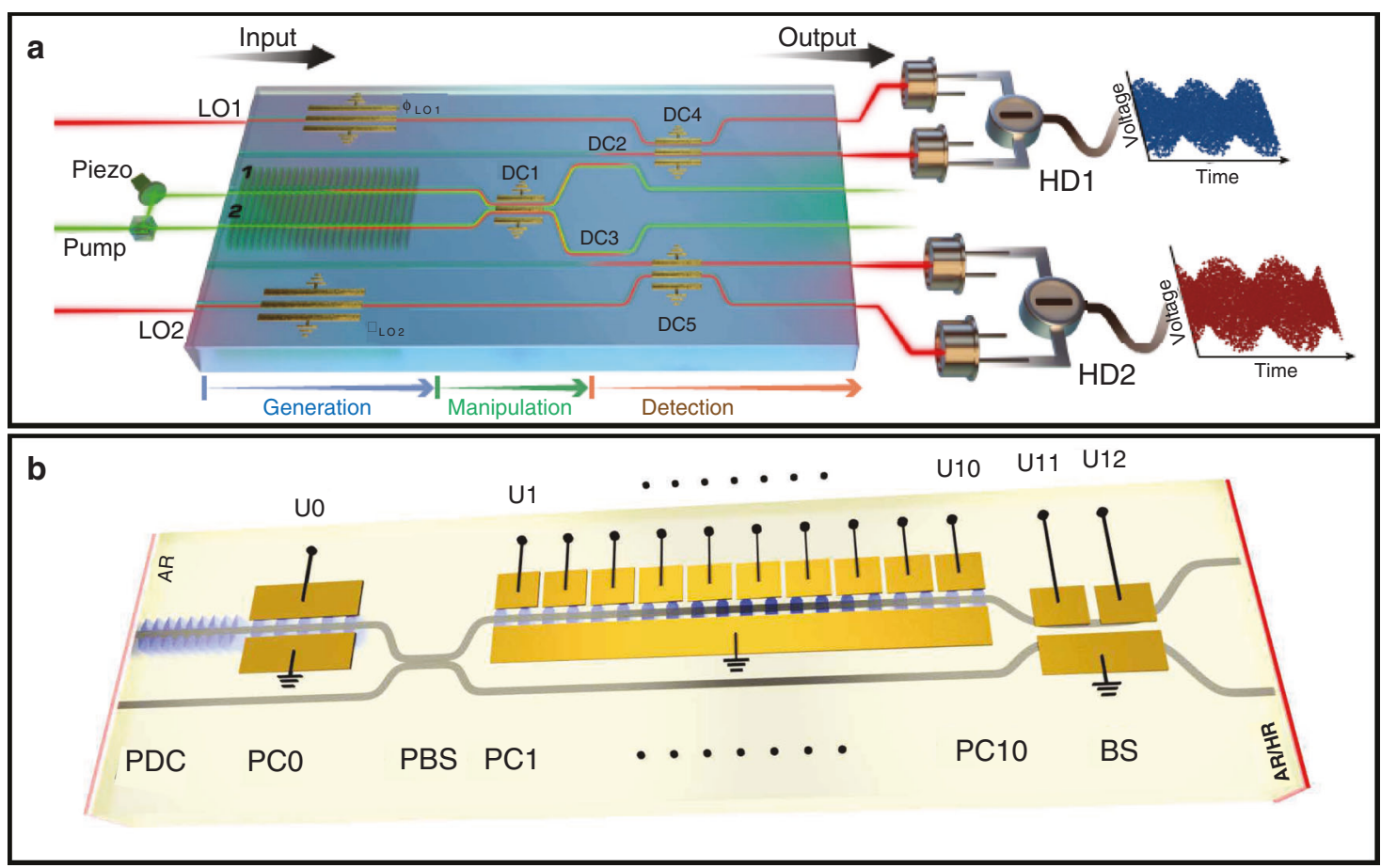

Fig. 9 a Schematic of integration with the generation, manipulation, and interferometric stages of homodyne detection of nonclassical light on a $\mathrm{H}$ : $\mathrm{LiNbO}_{3}$ waveguide chip ${ }^{129}$; Copyright 2018, American Association for the Advancement of Science. $\mathbf{b}$ Schematic of integration with the photon-pair generation, propagation, electro-optical path routing, and voltage-controllable time delay on a single Ti:LiNbO 3 waveguide chip ${ }^{130}$; Copyright 2019, American Association for the Advancement of Science

of 3-5 V. This exciting progress indicates that LN modulators are likely to be manufactured in the mid to long term. On the other hand, PPLN waveguides have been widely applied in nonlinear optics, while domain engineering of LNOI has emerged as a new branch of nonlinear integrated photonics. Nonlinear photonics plays a very important role in quantum technologies, which comprise an emerging class of devices capable of controlling the superposition and entanglement of quantum states of light, to realize fundamental performance advantages over ordinary classical devices. The entanglement photon-pair source and single-photon detector are very important components of quantum technologies, which can be achieved through nonlinear integrated photonics on LNOI. Considering the low-loss propagation waveguide and high-bandwidth integrated modulators achieved on LNOI, LNOI will be a promising matrix for integrated quantum technologies, with the entanglement photon-pair source and photon detector integrated on an optical chip (Fig. 9a, b) ${ }^{129-133}$.

Thus, we predict that integrated quantum photonics will enable the generation, processing, and detection of quantum states of light at increasing scale and level of complexity. Integrated quantum photonics can provide programmable devices approaching 1000 components occupying only millimeter-scale footprints with the integrated generation of multiphoton states. For instance, quantum computers are suggested to be the most remarkable and powerful future quantum technology ${ }^{134}$. The nonlinear integrated photonics approaches for singlephoton sources and detectors in optical quantum computers will be determined by their efficacy and practicality $^{131}$. Quantum communication aims to realize quantum key distribution (QKD) transmitters and receivers based on the laws of quantum mechanics for security, which is inaccessible by traditional communications ${ }^{135,136}$. On-chip integration with necessary components, such as tunable lasers, attenuators, and electrooptic phase modulators, can lead to the realization of QKD transmitters, while on-chip integration with singlephoton detectors and phase shifters can lead to the realization of QKD receivers.

Last, topological photonics may become an important application branch in integrated lithium niobate photonics. The topology, an indispensable degree of freedom of photonic systems that characterize the quantized global behavior of wavefunctions, can be optimized ${ }^{137,138}$. Topological effects can be realized in photonic crystals, coupled resonators, metamaterials and quasicrystals. Silicon photonics has been widely used to study topological features, whereas arrays of coupled optical resonators provide a toolbox to observe the wavefunction ${ }^{139,140}$. 
A "photonic molecule" with two distinct energy levels has been demonstrated using coupled microring resonators on LNOI, which can be controlled by external microwave excitation ${ }^{141}$. We predict that dynamically controlled multilevel photonic systems will promote research on topological photonics. Therefore, topological photonics on LNOI will open a new avenue for designing and controlling the behavior of light and possibly provide revolutionary applications.

The high quality and homogeneity of LN films are undoubtedly the most fundamental requirements for any photonic application. At present, the smart cut method is the main approach to prepare a submicron-thickness LN film. This method cannot meet the demands of large-scale manufacturing yet owing to the ion-implantation-induced defects and production efficiency. Therefore, the preparation approach of LNOI still needs to be improved and explored. The machine thinning method was also reported for the preparation of $\mathrm{LN}$ films ${ }^{142}$. The $\mathrm{LN}$ wafer can be polished to micron-scale and even submicron-scale films after bonding to $\mathrm{Si}$ substrates. It was reported that a micron-scale LN film was fabricated by this machine thinning method. An LN ridge waveguide with a crosssection of $1.3 \mu \mathrm{m} \times 4 \mu \mathrm{m}$ was fabricated on this film ${ }^{143}$. With an electric field applied along the z-axis, electrooptic modulation was demonstrated with a $V_{\pi} L$ of $7.1 \mathrm{~V} \mathrm{~cm}$, which is less than half that of a bulk LN modulator ${ }^{142}$. An LN modulator integrated onto a Si substrate using a CMOS-compatible process exhibited a frequency response of up to $110 \mathrm{GHz}^{144}$. First, the thinner the machined LN wafer is, the higher the confinement achieved by the LN ridge waveguides. Second, the machine thinning method does not involve ionimplantation-induced defects. Thus, the machine thinning method should be improved, and submicron LN films will be fabricated through this method. Thus, the machine thinning method may be an alternative approach for fabricating high-quality LN films.

Until now, LN films have generally been exfoliated from nondoped congruent LN crystals. The congruent LN crystals have a large coercive field $\left(21 \mathrm{kV} \mathrm{mm}^{-1}\right)$ and a low optical damage threshold because of intrinsic defects $^{145}$. An electric field of up to $40 \mathrm{kV} \mathrm{mm}^{-1}$ is needed to invert the ferroelectric domain in an $\mathrm{x}$-cut $\mathrm{LN}$ film for QPM nonlinear integrated photonics. Therefore, an Mg-doped LN film was adopted in integrated photonics, which reduced the poling electric field to $7.6 \mathrm{kV} \mathrm{mm}^{-1}$. The waveguide showed no photorefractive damage at a high $\mathrm{SH}$ optical intensity of $\sim 10 \mathrm{MW} \mathrm{\textrm {cm } ^ { - 2 }}$ after many hours of optical pumping ${ }^{120}$. Furthermore, stoichiometric LN crystals have a smaller coercive field of $\sim 3.5 \mathrm{kV} \mathrm{mm}^{-1}$ and a completely crystalline structure ${ }^{146-152}$. Therefore, stoichiometric LN films should be more suitable for ultralow-loss nanophotonic waveguides ${ }^{153}$ and can be poled with a smaller electric field.

Over the last decade, integrated lithium niobate photonics has been rapidly developed and proven invaluable in the development of future optical communication and quantum technologies. The large-scale and low-cost manufacturing of integrated photonic devices and systems by mature manufacturing processes will enable new revolutionary applications in optical communication and quantum technologies.

\section{Acknowledgements}

The authors are thankful for the funding from the National Natural Science Foundation of China (Grant nos. 51802113 and 51802116), High Technology Research and Development Program of Shandong Province (Grant no. 2018***0209), and the Natural Science Foundation of Shandong Province (Grant nos. ZR2018BEM015 and ZR2019LLZ003).

\section{Author details}

${ }^{1}$ Collaborative Innovation Center of Technology and Equipment for Biological Diagnosis and Therapy in Universities of Shandong, Institute for Advanced Interdisciplinary Research (iAIR), University of Jinan, Jinan 250022, China. ${ }^{2}$ Jinan Institute of Quantum Technology, Jinan 250101, China. ${ }^{3}$ CETC Deqing Huaying Electronics Co., Ltd., Huzhou 313200, China. ${ }^{4}$ Crystrong Photoelectric

Technology Co., Ltd., Jinan 250100, China. ${ }^{5}$ State Key Laboratory of Crystal Materials, Shandong University, Jinan 250100, China

\section{Conflict of interest}

The authors declare that they have no conflict of interest.

Received: 10 May 2020 Revised: 8 November 2020 Accepted: 12 November 2020

Published online: 10 December 2020

\section{References}

1. Imran, M. et al. A survey of optical carrier generation techniques for terabit capacity elastic optical networks. IEEE Commun. Surv. Tutor. 20, 211-263 (2018).

2. Temprana, E. et al. Overcoming Kerr-induced capacity limit in optical fiber transmission. Science 348, 1445-1448 (2015).

3. Hecht, J. The bandwidth bottleneck that is throttling the Internet. Nature 536, 139-142 (2016)

4. Capmany, J. \& Novak, D. Microwave photonics combines two worlds. Nat. Photonics 1, 319-330 (2007).

5. Yao, J. P. Microwave photonics. J. Lightwave Technol. 27, 314-335 (2009).

6. Osgood, R. Integrated optics: slice of perfection. Nat. Photonics 1, 365-366 (2007).

7. Wooten, E. L. et al. A review of lithium niobate modulators for fiber-optic communications systems. IEEE J. Sel. Top. Quantum Electron. 6, 69-82 (2000).

8. Bazzan, M. \& Sada, C. Optical waveguides in lithium niobate: recent developments and applications. Appl. Phys. Rev. 2, 040603 (2015).

9. Wang, T. X. et al. Periodically poled $\mathrm{LiNbO}_{3}$ crystals from $1 \mathrm{D}$ and $2 \mathrm{D}$ to $3 \mathrm{D}$. Sci. China Technol. Sci. 63, 1110-1126 (2020).

10. Wei, D. Z. et al. Efficient nonlinear beam shaping in three-dimensional lithium niobate nonlinear photonic crystals. Nat. Commun. 10, 4193 (2019).

11. $\mathrm{Hu}, \mathrm{X}$. P., Xu, P. \& Zhu, S. N. Engineered quasi-phase-matching for laser techniques [Invited]. Photonics. Photonics Res. 1, 171-185 (2013).

12. Zhu, S. N., Zhu, Y. Y. \& Ming, N. B. Quasi-phase-matched third-harmonic generation in a quasi-periodic optical superlattice. Science 278, 843-846 (1997).

13. Wang, D. Z. et al. Three-wavelength green laser using intracavity frequency conversion of $\mathrm{Nd}: \mathrm{Mg}_{\mathrm{LiTaO}}$ with a MgO:PPLN crystal. Appl. Phys. B 117, 1117-1121 (2014).

14. Liu, H. Y. et al. $1514 \mathrm{~nm}$ eye-safe passively Q-switched self-optical parametric oscillator based on $\mathrm{Nd}_{3}^{+}$-doped MgO:PPLN. Chin. Opt. Lett. 17, 111404 (2019). 
15. Wang, D. Z. et al. Periodically poled self-frequency-doubling green laser fabricated from Nd:Mg:LiNbO 3 single crystal. Opt. Express 23, 17727-17738 (2015).

16. Shoji, I. et al. Absolute scale of second-order nonlinear-optical coefficients. J. Opt. Soc. Am. B 14, 2268-2294 (1997).

17. Marpaung, D. et al. Integrated microwave photonics. Laser Photonics Rev. 7 506-538 (2013).

18. Marpaung, D., Yao, J. P. \& Capmany, J. Integrated microwave photonics. Nat. Photonics 13, 80-90 (2019).

19. Eggleton, B. J. et al. Brillouin integrated photonics. Nat. Photonics 13, 664-677 (2019).

20. Cassan, E. et al. Nonlinear integrated photonics. Photonics Res. 6, NIP1-NIP2 (2018)

21. Wilson, D. J. et al. Integrated gallium phosphide nonlinear photonics. Nat. Photonics 14, 57-62 (2020).

22. Oser, D. et al. High-quality photonic entanglement out of a stand-alone silicon chip. npj Quantum Inf. 6, 31 (2020).

23. Li, A. \& Bogaerts, W. Reconfigurable nonlinear nonreciprocal transmission in a silicon photonic integrated circuit. Optica 7, 7-14 (2020).

24. Smit, M. et al. An introduction to InP-based generic integration technology Semicond. Sci. Technol. 29, 083001 (2014).

25. Moss, D. J. et al. New CMOS-compatible platforms based on silicon nitride and Hydex for nonlinear optics. Nat. Photonics 7, 597-607 (2013).

26. Leuthold, J., Koos, C. \& Freude, W. Nonlinear silicon photonics. Nat. Photonics 4, 535-544 (2010).

27. Jacobsen, R. S. et al. Strained silicon as a new electro-optic material. Nature 441, 199-202 (2006).

28. $\mathrm{Xu}, \mathrm{Q}$. F. et al. Micrometre-scale silicon electro-optic modulator. Nature $\mathbf{4 3 5}$ 325-327 (2005).

29. Roeloffzen, C. G. H. et al. Silicon nitride microwave photonic circuits. Opt. Express 21, 22937-22961 (2013).

30. Horikawa, K., Nakasuga, Y. \& Ogawa, H. Self-heterodyning optical waveguide beam forming and steering network integrated on Lithium Niobate substrate. IEEE Trans. Microw. Theory Tech. 43, 2395-2401 (1995).

31. Janner, D. et al. Micro-structured integrated electro-optic LiNbO3 modulators. Laser Photonics Rev. 3, 301-313 (2009).

32. Jalali, B. \& Fathpour, S. Silicon photonics. J. Lightwave Technol. 24, 4600-4615 (2006).

33. Fischer, $U$. et al. $0.1 \mathrm{~dB} / \mathrm{cm}$ waveguide losses in single-mode $\mathrm{SOI}$ rib waveguides. IEEE Photonics Technol. Lett. 8, 647-648 (1996).

34. Cox, C. H. et al. Limits on the performance of RF-over-fiber links and their impact on device design. IEEE Trans. Microw. Theory Tech. 54, 906-920 (2006).

35. Gardes, F. Y. et al. A sub-micron depletion-type photonic modulator in Silicon On Insulator. Opt. Express 13, 8845-8854 (2005).

36. Jin, $H$. et al. On-chip generation and manipulation of entangled photons based on reconfigurable lithium-niobate waveguide circuits. Phys. Rev. Lett. 113, 103601 (2014)

37. Boes, A. et al. Status and potential of lithium niobate on insulator (LNOI) for photonic integrated circuits. Laser Photonics Rev. 12, 1700256 (2018).

38. Lončar, M. Integrated lithium niobate photonics and applications. in Proc. Nonlinear Optics. (Optical Society of America, Waikoloa Beach, 2019). https:// www.osapublishing.org/abstract.cfm?URI=NLO-2019-NW3A.5.

39. Kong, Y. F. et al. Recent progress in lithium niobate: optical damage, defect simulation, and on-chip devices. Adv. Mater. 32, 1806452 (2020).

40. Qi, Y. F. \& Li, Y. Integrated lithium niobate photonics. Nanophotonics 9 , 1287-1320 (2020).

41. Poberaj, $G$. et al. Lithium niobate on insulator (LNOI) for micro-photonic devices. Laser Photonics Rev. 6, 488-503 (2012).

42. Wang, J. W. et al. Integrated photonic quantum technologies. Nat. Photonics 14, 273-284 (2020).

43. He, M. B. et al. High-performance hybrid silicon and lithium niobate Mach-Zehnder modulators for $100 \mathrm{Gbit} \mathrm{s}^{-1}$ and beyond. Nat. Photonics 13 359-364 (2019).

44. Heylman, K. D. et al. Optical microresonators for sensing and transduction: a materials perspective. Adv. Mater. 29, 1700037 (2017).

45. Rabiei, P. \& Gunter, P. Optical and electro-optical properties of submicrometer lithium niobate slab waveguides prepared by crystal ion slicing and wafer bonding. Appl. Phys. Lett. 85, 4603-4605 (2004).

46. Rabiei, P. \& Steier, W. H. Lithium niobate ridge waveguides and modulators fabricated using smart guide. Appl. Phys. Lett. 86, 161115 (2005).
47. Park, Y. B. et al. Integration of single-crystal LiNbO3 thin film on silicon by laser irradiation and ion implantation-induced layer transfer. Adv. Mater. 18 1533-1536 (2006).

48. Guarino, A. et al. Electro-optically tunable microring resonators in lithium niobate. Nat. Photonics 1, 407-410 (2007).

49. Poberaj, G. et al. Ion-sliced lithium niobate thin films for active photonic devices. Optical Mater. 31, 1054-1058 (2009).

50. $\mathrm{Hu}, \mathrm{H}$. et al. Lithium niobate photonic wires. in Proc. 2010 23rd Annual Meeting of the IEEE Photonics Society. 254-255 (IEEE, Denver, USA, 2010).

51. Wang, J. et al. High-Q lithium niobate microdisk resonators on a chip for efficient electro-optic modulation. Opt. Express 23, 23072-23078 (2015).

52. Ulliac, G. et al. Argon plasma inductively coupled plasma reactive ion etching study for smooth sidewall thin film lithium niobate waveguide application Opt. Mater. 53, 1-5 (2016).

53. Wang, C. et al. Second harmonic generation in nano-structured thin-film lithium niobate waveguides. Opt. Express 25, 6963-6973 (2017).

54. Wang, C. et al. Nanophotonic lithium niobate electro-optic modulators. Opt. Express 26, 1547-1555 (2018).

55. Zhang, M. et al. Monolithic ultra-high-Q lithium niobate microring resonator. Optica 4, 1536-1537 (2017).

56. Wang, C. et al. Integrated lithium niobate electro-optic modulators operating at CMOS-compatible voltages. Nature 562, 101-104 (2018).

57. Rao, A. et al. High-performance and linear thin-film lithium niobate MachZehnder modulators on silicon up to $50 \mathrm{GHz}$. Opt Lett. 41, 5700-5703 (2016).

58. Mercante, A. J. et al. Thin film lithium niobate electro-optic modulator with terahertz operating bandwidth. Opt. Express 26, 14810-14816 (2018).

59. Lee, Y. S. et al. Hybrid $\mathrm{Si}_{\mathrm{L}} \mathrm{iNbO}_{3}$ microring electro-optically tunable resonators for active photonic devices. Opt. Lett. 36, 1119-1121 (2011).

60. Chen, L. \& Reano, R. M. Compact electric field sensors based on indirect bonding of lithium niobate to silicon microrings. Opt. Express 20, 4032-4038 (2012).

61. Chen, L., Wood, M. G. \& Reano, R. M. 12.5 pm/ hybrid silicon and lithium niobate optical microring resonator with integrated electrodes. Opt. Express 21, 27003-27010 (2013).

62. Chen, L. et al. Hybrid silicon and lithium niobate electro-optical ring modulator. Optica 1, 112-118 (2014).

63. Weigel, P. O. et al. Bonded thin film lithium niobate modulator on a silicon photonics platform exceeding $100 \mathrm{GHz} 3-\mathrm{dB}$ electrical modulation bandwidth. Opt. Express 26, 23728-23739 (2018).

64. Xie, W. Q. et al. On-chip integrated quantum-dot-silicon-nitride microdisk lasers. Adv. Mater. 29, 1604866 (2017).

65. Chang, L. et al. Heterogeneous integration of lithium niobate and silicon nitride waveguides for wafer-scale photonic integrated circuits on silicon. Opt. Lett. 42, 803-806 (2017).

66. Ahmed, A. N. R. et al. Vertical mode transition in hybrid lithium niobate and silicon nitride-based photonic integrated circuit structures. Opt. Lett. 43, 4140-4143 (2018).

67. Rabiei, P. et al. Heterogeneous lithium niobate photonics on silicon substrates. Opt. Express 21, 25573-25581 (2013).

68. Rabiei, P. et al. Submicron optical waveguides and microring resonators fabricated by selective oxidation of tantalum. Opt. Express 21, 6967-6972 (2013).

69. Rao, A. et al. Heterogeneous microring and Mach-Zehnder modulators based on lithium niobate and chalcogenide glasses on silicon. Opt. Express 23, 22746-22752 (2015).

70. Chiles, J. \& Fathpour, S. Mid-infrared integrated waveguide modulators based on silicon-on-lithium-niobate photonics. Optica 1, 350-355 (2014).

71. Jin, S. L. et al. LiNbO 3 thin-film modulators using silicon nitride surface ridge waveguides. IEEE Photonics Technol. Lett. 28, 736-739 (2016).

72. Ahmed, A. N. R. et al. Tunable hybrid silicon nitride and thin-film lithium niobate electro-optic microresonator. Opt. Lett. 44, 618-621 (2019).

73. Ahmed, A. N. R. et al. High-performance racetrack resonator in silicon nitride -thin film lithium niobate hybrid platform. Opt. Express 27, 30741-30751 (2019).

74. Marpaung, D. et al. Nonlinear integrated microwave photonics. J. Lightwave Technol. 32, 3421-3427 (2014).

75. Hendrickson, S. M. et al. Integrated nonlinear photonics: emerging applications and ongoing challenges [Invited]. J. Opt. Soc. Am. B 31, 3193-3203 (2014).

76. Caspani, L. et al. Optical frequency conversion in integrated devices [Invited] J. Opt. Soc. Am. B 28, A67-A82 (2011). 
77. Autere, A. et al. Nonlinear optics with 2D layered materials. Adv. Mater. 30, 1705963 (2018).

78. Helmy, A. S. et al. Recent advances in phase matching of second-order nonlinearities in monolithic semiconductor waveguides. Laser Photonics Rev. 5, 272-286 (2011).

79. DeSalvo, R. et al. Infrared to ultraviolet measurements of two-photon absorption and n/sub 2/ in wide bandgap solids. IEEE J. Quantum Electron. 32, 1324-1333 (1996).

80. Bi, Z. F. et al. High-efficiency second-harmonic generation in doubly-resonant $X^{(2)}$ microring resonators. Opt. Express 20, 7526-7543 (2012).

81. Kauranen, M. \& Zayats, A. V. Nonlinear plasmonics. Nat. Photonics 6, 737-748 (2012).

82. Zhao, Y. et al. Visible nonlinear photonics via high-order-mode dispersion engineering. Optica 7, 135-141 (2020).

83. Vahala, K. J. Optical microcavities. Nature 424, 839-846 (2003).

84. $\mathrm{Wu}, \mathrm{J}$. Y. et al. $2 \mathrm{D}$ layered graphene oxide films integrated with micro-ring resonators for enhanced nonlinear optics. Small 16, 1906563 (2020).

85. Mi, Y. et al. High-quality hexagonal nonlayered CdS nanoplatelets for lowthreshold whispering-gallery-mode lasing. Small 15, 1901364 (2019).

86. Wang, C. et al. Integrated high quality factor lithium niobate microdisk resonators. Opt. Express 22, 30924-30933 (2014).

87. Luo, R. et al. On-chip second-harmonic generation and broadband parametric down-conversion in a lithium niobate microresonator. Opt. Express $\mathbf{2 5}$ 24531-24539 (2017)

88. Wolf, R. et al. Cascaded second-order optical nonlinearities in on-chip micro rings. Opt. Express 25, 29927-29933 (2017).

89. Yu, M. J. et al. Raman lasing and soliton mode-locking in lithium niobate microresonators. Light 9, 9 (2020).

90. Kippenberg, T. J., Holzwarth, R. \& Diddams, S. A. Microresonator-based optical frequency combs. Science 332, 555-559 (2011).

91. Torres-Company, V. \& Weiner, A. M. Optical frequency comb technology for ultra-broadband radio-frequency photonics. Laser Photonics Rev. 8, 368-393 (2014).

92. Chang, L. et al. Ultra-efficient frequency comb generation in AlGaAs-oninsulator microresonators. Nat. Commun. 11, 1331 (2020).

93. Holzwarth, R. et al. Optical frequency synthesizer for precision spectroscopy. Phys. Rev. Lett. 85, 2264-2267 (2000)

94. Liang, W. et al. High spectral purity Kerr frequency comb radio frequency photonic oscillator. Nat. Commun. 6, 7957 (2015).

95. Kippenberg, T. J. et al. Dissipative Kerr solitons in optical microresonators. Science 361, eaan8083 (2018).

96. Pfeifle, J. et al. Coherent terabit communications with microresonator Kerr frequency combs. Nat. Photonics 8, 375-380 (2014).

97. Del'Haye, P. et al. Optical frequency comb generation from a monolithic microresonator. Nature 450, 1214-1217 (2007).

98. Wang, C. et al. Monolithic lithium niobate photonic circuits for Kerr frequency comb generation and modulation. Nat. Commun. 10, 978 (2019).

99. Beha, K. et al. Electronic synthesis of light. Optica 4, 406-411 (2017).

100. Soltani, M. et al. Efficient quantum microwave-to-optical conversion using electro-optic nanophotonic coupled resonators. Phys. Rev. A 96, 043808 (2017).

101. Rueda, A. et al. Resonant electro-optic frequency comb. Nature $\mathbf{5 6 8}, \mathbf{3 7 8 - 3 8 1}$ (2019)

102. Zhang, M. et al. Broadband electro-optic frequency comb generation in a lithium niobate microring resonator. Nature 568, 373-377 (2019).

103. Hanke, T. et al. Tailoring spatiotemporal light confinement in single plasmonic nanoantennas. Nano Lett. 12, 992-996 (2012).

104. Husu, H. et al. Metamaterials with tailored nonlinear optical response. Nano Lett. 12, 673-677 (2012).

105. Sain, B. \& Zentgraf, T. Metasurfaces help lasers to mode-lock. Light 9, 67 (2020).

106. Neshev, D. \& Aharonovich, I. Optical metasurfaces: new generation building blocks for multi-functional optics. Light 7, 58 (2018).

107. Wang, C. et al. Metasurface-assisted phase-matching-free second harmonic generation in lithium niobate waveguides. Nat. Commun. 8, 2098 (2017).

108. Moutzouris, K. et al. Second-harmonic generation through optimized modal phase matching in semiconductor waveguides. Appl. Phys. Lett. 83, 620-622 (2003).

109. Chen, J. Y. et al. Modal phase matched lithium niobate nanocircuits for integrated nonlinear photonics. OSA Contin. 1, 229-242 (2018).
110. Geiss, R. et al. Fabrication of nanoscale lithium niobate waveguides for second-harmonic generation. Opt. Lett. 40, 2715-2718 (2015).

111. Luo, R. et al. Highly tunable efficient second-harmonic generation in a lithium niobate nanophotonic waveguide. Optica 5, 1006-1011 (2018).

112. Hayat, A. et al. Phasematching in semiconductor nonlinear optics by linear long-period gratings. Appl. Phys. Lett. 92, 181110 (2008).

113. Somekh, S. \& Yariv, A. Phase-matchable nonlinear optical interactions in periodic thin films. Appl. Phys. Lett. 21, 140-141 (1972).

114. Rao, A. et al. Second-harmonic generation in single-mode integrated waveguides based on mode-shape modulation. Appl. Phys. Lett. 110, 111109 (2017).

115. Gainutdinov, R. V., Volk, T. R. \& Zhang, H. H. Domain formation and polarization reversal under atomic force microscopy-tip voltages in ionsliced $\mathrm{LiNbO}_{3}$ films on $\mathrm{SiO}_{2} / \mathrm{LiNbO}_{3}$ substrates. Appl. Phys. Lett. 107, 162903 (2015).

116. Mackwitz, P. et al. Periodic domain inversion in x-cut single-crystal lithium niobate thin film. Appl. Phys. Lett. 108, 152902 (2016).

117. Rao, A. et al. Second-harmonic generation in periodically-poled thin film lithium niobate wafer-bonded on silicon. Opt. Express 24, 29941-29947 (2016).

118. Chang, L. et al. Thin film wavelength converters for photonic integrated circuits. Optica 3, 531-535 (2016).

119. Stanicki, B. J. et al. Surface domain engineering in lithium niobate. OSA Contin. 3, 345-358 (2020).

120. Wang, C. et al. Ultrahigh-efficiency wavelength conversion in nanophotonic periodically poled lithium niobate waveguides. Optica 5, 1438-1441 (2018).

121. Rao, A. et al. Actively-monitored periodic-poling in thin-film lithium niobate photonic waveguides with ultrahigh nonlinear conversion efficiency of 4600 $\% \mathrm{~W}^{-1} \mathrm{~cm}^{-2}$. Opt. Express 27, 25920-25930 (2019).

122. Li, G. Z. et al. Broadband sum-frequency generation using $d_{33}$ in periodically poled $\mathrm{LiNbO}_{3}$ thin film in the telecommunications band. Opt. Lett. 42 939-942 (2017).

123. Liang, L. Y. et al. Facile approach for the periodic poling of MgO-doped lithium niobate with liquid electrodes. CrystEngComm 21, 941-947 (2019).

124. Wolf, R. et al. Quasi-phase-matched nonlinear optical frequency conversion in on-chip whispering galleries. Optica 5, 872-875 (2018).

125. Hao, Z. Z. et al. Second-harmonic generation using $d_{33}$ in periodically poled lithium niobate microdisk resonators. Photonics Res. 8, 311-317 (2020).

126. Lin, J. T. et al. Broadband quasi-phase-matched harmonic generation in an on-chip monocrystalline lithium niobate microdisk resonator. Phys. Rev. Lett. 122, 173903 (2019).

127. Ilchenko, V. S. et al. Nonlinear optics and crystalline whispering gallery mode cavities. Phys. Rev. Lett. 92, 043903 (2004).

128. Lu, J. J. et al. Periodically poled thin-film lithium niobate microring resonators with a second-harmonic generation efficiency of 250,000\%/W. Optica 6, 1455-1460 (2019).

129. Lenzini, F. et al. Integrated photonic platform for quantum information with continuous variables. Sci. Adv. 4, eaat9331 (2018).

130. Luo, K. H. et al. Nonlinear integrated quantum electro-optic circuits. Sci. Adv. 5, eaat1451 (2019).

131. O'Brien, J. L. Optical quantum computing. Science 318, 1567-1570 (2007).

132. Flöry, N. et al. Waveguide-integrated van der Waals heterostructure photodetector at telecom wavelengths with high speed and high responsivity. Nat. Nanotechnol. 15, 118-124 (2020).

133. Caspani, L. et al. Integrated sources of photon quantum states based on nonlinear optics. Light 6, e17100 (2017).

134. Ríos, C. et al. In-memory computing on a photonic platform. Sci. Adv. 5, eaau5759 (2019).

135. Pan, J. W. et al. Entanglement purification for quantum communication. Nature 410, 1067-1070 (2001).

136. Yu, Y. et al. Entanglement of two quantum memories via fibres over dozens of kilometres. Nature 578, 240-245 (2020).

137. Lu, L., Joannopoulos, J. D. \& Soljačić, M. Topological photonics. Nat. Photonics 8, 821-829 (2014).

138. $\mathrm{Xu}, \mathrm{X}$. Y. et al. Measuring a dynamical topological order parameter in quantum walks. Light 9, 7 (2020).

139. Lin, Q. et al. Photonic Weyl point in a two-dimensional resonator lattice with a synthetic frequency dimension. Nat. Commun. 7, 13731 (2016).

140. Hafezi, M. et al. Imaging topological edge states in silicon photonics. Nat. Photonics 7, 1001-1005 (2013). 
141. Zhang, M. et al. Electronically programmable photonic molecule. Nat. Photonics 13, 36-40 (2019).

142. Macario, J. et al. Full spectrum millimeter-wave modulation. Opt. Express 20, 23623-23629 (2012).

143. Mercante, A. J. et al. Thin $\mathrm{LiNbO}_{3}$ on insulator electro-optic modulator. Opt. Lett. 41, 867-869 (2016).

144. Mercante, A. J. et al. $110 \mathrm{GHz}$ CMOS compatible thin film $\mathrm{LiNbO}_{3}$ modulator on silicon. Opt. Express 24, 15590-15595 (2016).

145. Sun, D. H. et al. Origin of ferroelectric modification: the thermal behavior of dopant ions. Cryst. Growth Des. 18, 4860-4863 (2018).

146. Kitamura, K. et al. Stoichiometric $\mathrm{LiNbO}_{3}$ single crystal growth by double crucible Czochralski method using automatic powder supply system. J. Cryst. Growth 116, 327-332 (1992).

147. Yan, $\mathrm{T}$. et al. Growth of $\mathrm{MgO}$ doped near stoichiometric $\mathrm{LiNbO}_{3}$ single crystals by a hanging crucible Czochralski method using a ship lockage type powder feeding system assisted by numerical simulation. CrystEngComm 16, 6593-6602 (2014).
148. Wang, F. L. et al. Yb sensitized near-stoichiometric Er:LiNbO 3 single crystal: a matrix for optical communication and upconversion emission. Cryst. Growth Des. 18, 1495-1500 (2018).

149. Kang, X. L. et al. Formation mechanism and elimination methods for anti-site defects in $\mathrm{LiNbO}_{3} / \mathrm{LiTaO}_{3}$ crystals. CrystEngComm 18, 8136-8146 (2016).

150. Sun, D. et al. Antisite defect elimination through $\mathrm{Mg}$ doping in stoichiometric lithium tantalate powder synthesized via a wet-chemical spray-drying method. J. Appl. Crystallogr. 48, 377-385 (2015).

151. Yao, S. H. et al. Growth, optical and thermal properties of near-stoichiometric $\mathrm{LiNbO}_{3}$ single crystal. J. Alloy. Compd. 455, 501-505 (2008).

152. Wang, L. et al. Optical waveguide in stoichiometric lithium niobate formed by 500 keV proton implantation. Opt. Express 15, 16880-16885 (2007).

153. Cai, L. T. et al. Integrated optics on single-crystal lithium niobate thin film: some recent progress. in Proc. 2016 18th International Conference on Transparent Optical Networks. 1-4 (IEEE, Trento 2016).

154. Hu, H., Ricken, R. \& Sohler, W. Lithium niobate photonic wires. Opt. Express 17, 24261-24268 (2009). 